\title{
Roughness perception of unfamiliar dot pattern textures
}

Citation for published version (APA):

Eck, J., Kaas, A. L., Mulders, J. L. J., \& Goebel, R. (2013). Roughness perception of unfamiliar dot pattern textures. Acta Psychologica, 143(1), 20-34. https://doi.org/10.1016/j.actpsy.2013.02.002

Document status and date:

Published: 01/05/2013

DOI:

10.1016/j.actpsy.2013.02.002

Document Version:

Publisher's PDF, also known as Version of record

Document license:

Taverne

Please check the document version of this publication:

- A submitted manuscript is the version of the article upon submission and before peer-review. There can be important differences between the submitted version and the official published version of record.

People interested in the research are advised to contact the author for the final version of the publication, or visit the DOI to the publisher's website.

- The final author version and the galley proof are versions of the publication after peer review.

- The final published version features the final layout of the paper including the volume, issue and page numbers.

Link to publication

\footnotetext{
General rights rights.

- You may freely distribute the URL identifying the publication in the public portal. please follow below link for the End User Agreement:

www.umlib.nl/taverne-license

Take down policy

If you believe that this document breaches copyright please contact us at:

repository@maastrichtuniversity.nl

providing details and we will investigate your claim.
}

Copyright and moral rights for the publications made accessible in the public portal are retained by the authors and/or other copyright owners and it is a condition of accessing publications that users recognise and abide by the legal requirements associated with these

- Users may download and print one copy of any publication from the public portal for the purpose of private study or research.

- You may not further distribute the material or use it for any profit-making activity or commercial gain

If the publication is distributed under the terms of Article $25 \mathrm{fa}$ of the Dutch Copyright Act, indicated by the "Taverne" license above, 


\title{
Roughness perception of unfamiliar dot pattern textures ${ }^{\text {}}$
}

\author{
Judith Eck a,b,*, Amanda L. Kaas ${ }^{\text {a }}$, Joost L.J. Mulders ${ }^{\text {b }}$, Rainer Goebel ${ }^{\text {a,b }}$ \\ a Maastricht University, Department of Cognitive Neuroscience, 6229 ER Maastricht, The Netherlands \\ b Brain Innovation B.V., 6229 EV Maastricht, The Netherlands
}

\section{A R T I C L E I N F O}

\section{Article history:}

Received 13 September 2012

Received in revised form 10 January 2013

Accepted 2 February 2013

Available online 20 March 2013

\section{PsycINFO classification:}

2320

Keywords:

Texture perception

Roughness estimation

Stimulus familiarity

Sensory modality

\begin{abstract}
A B S T R A C T
Both vision and touch yield comparable results in terms of roughness estimation of familiar textures as was shown in earlier studies. To our knowledge, no research has been conducted on the effect of sensory familiarity with the stimulus material on roughness estimation of unfamiliar textures.

The influence of sensory modality and familiarity on roughness perception of dot pattern textures was investigated in a series of five experiments. Participants estimated the roughness of textures varying in mean center-to-center dot spacing in experimental conditions providing visual, haptic and visual-haptic combined information.

The findings indicate that roughness perception of unfamiliar dot pattern textures is well described by a bi-exponential function of inter-dot spacing, regardless of the sensory modality used. However, sensory modality appears to affect the maximum of the psychophysical roughness function, with visually perceived roughness peaking for a smaller inter-dot spacing than haptic roughness. We propose that this might be due to the better spatial acuity of the visual modality. Individuals appeared to use different visual roughness estimation strategies depending on their first sensory experience (visual vs. haptic) with the stimulus material, primarily in an experimental context which required the combination of visual and haptic information in a single bimodal roughness estimate. Furthermore, the similarity of findings in experimental settings using real and virtual visual textures indicates the suitability of the experimental setup for neuroimaging studies, creating a more direct link between behavioral and neuroimaging results.
\end{abstract}

(c) 2013 Elsevier B.V. All rights reserved.

\section{Introduction}

Texture as a surface property plays a crucial role in object identification and recognition (Lacey, Hall, \& Sathian, 2010) as well as in interaction with objects in our environment. We can tell peaches and nectarines apart not because of the color or the geometry but because of their different surface textures. Texture perception has been predominantly investigated in the haptic domain as the haptic sense appears to be dominant in perceiving material properties (Klatzky, Lederman, \& Reed, 1987). However, our visual and auditory senses also process texture information. Roughness, in particular, can be distinguished not only by touch but also by the different looks of rough and smooth textures (Bergmann Tiest \& Kappers, 2007) and by the different sounds that are generated when touching those surfaces (Guest, Catmur, Lloyd, \& Spence, 2002).

\footnotetext{
The authors would like to point out that the first experiment has been published in the following conference paper: Eck, J., Kaas, A. L., \& Goebel R. (2011). The effect of sensory modality and previous experience on perceived roughness. Proceedings IEEE World Haptics Conference, 209-214.

* Corresponding author at: Maastricht University, Department of Cognitive Neuroscience, 6229 ER Maastricht, The Netherlands. Tel.: + 3143 2100120; fax: + 31432100121.

E-mail address: judith.eck@maastrichtuniversity.nl (J. Eck).
}

It is important to differentiate roughness as a perceptual quality from the physical characteristics that evoke it. In the present study, we will refer to perceived roughness to stress the subjective character. Roughness as a physical concept was previously defined as a measure of height differences combined with spatial properties of the surface (Bergmann Tiest \& Kappers, 2006). At the level of sensory neurons, haptic roughness perception is mainly mediated by a channel sensitive to vibratory information and a channel sensitive to spatial variance, as already hypothesized by Katz in 1925 (Katz, 1989). Evidence for this so-called duplex model of (tactual) roughness perception was found in a number of experiments by Hollins and colleagues (Bensmaïa \& Hollins, 2005; Hollins \& Risner, 2000). The authors reported that roughness perception was crucially dependent on vibrotactile cues for particle sizes below $100 \mu \mathrm{m}$. The spatial pressure distribution was more important for coarser textures, for which the roughness percept was highly correlated with variation in firing rate of SA1 afferents (Blake, Hsiao, \& Johnson, 1997; Connor, Hsiao, Phillips, \& Johnson, 1990).

Perceived roughness is influenced by physical and physiological as well as context parameters (see Bergmann Tiest, 2010). The literature is rich in studies investigating these influences, though primarily in the haptic domain. A recent review by Bergmann Tiest (2010) concluded that tactual roughness perception is associated with physical surface properties such as height difference, friction, spatial period 
and dot spacing. Both natural familiar textures, like fabrics, wood, foams and glass (Bergmann Tiest \& Kappers, 2006; Yoshioka, Bensmaïa, Craig, \& Hsiao, 2007) and unfamiliar textures like linear gratings (Cascio \& Sathian, 2001; Lawrence, Kitada, Klatzky, \& Lederman, 2007) and dot pattern stimuli (Dépeault, Meftah, \& Chapman, 2009; Kahrimanovic, Bergmann Tiest, \& Kappers, 2009; Klatzky \& Lederman, 1999; Lederman, Klatzky, Hamilton, \& Ramsay, 1999) have been used in a wide range of experiments investigating roughness perception. The current study employed dot pattern stimuli, because they have the advantage that one dimension can be varied parametrically while keeping all other dimensions constant. An additional advantage of (isotropic) dot patterns is that the evoked percept is independent of the haptic exploration direction. In previous studies, increasing dot diameter resulted in a decreased haptic roughness percept (Blake et al., 1997; Connor et al., 1990), whereas dot height influenced perceived roughness only for dot patterns with rather small dot diameters (e.g., 0.25-1.60 mm) (Blake et al., 1997). (Ir)regularity of the pattern (Dépeault et al., 2009) as well as scanning speed of the textures (Meftah, Belingard, \& Chapman, 2000) did not affect roughness perception with the bare finger. The main factor contributing to roughness perception of these dot patterns appeared to be the mean inter-element spacing of the textures, with an increased roughness percept for increasing inter-dot spacing (Connor et al., 1990; Dépeault et al., 2009; Gescheider, Bolanowski, Greenfield, \& Brunette, 2005; Lederman, Thorne, \& Jones, 1986; Meftah et al., 2000; Merabet et al., 2004, 2007). However, it is still a matter of debate whether this psychophysical function is more or less linear (Dépeault et al., 2009; Meftah et al., 2000) or rather represented by an inverted U-shape peaking at about $3 \mathrm{~mm}$ inter-dot spacing (Connor et al., 1990; Gescheider et al., 2005; Merabet et al., 2004, 2007). The curves depicted in these latter studies suggest that the perceived roughness function has a steep positive slope for interelement spacings below $3 \mathrm{~mm}$ and a shallower negative slope above $3 \mathrm{~mm}$, indicating that a simple symmetric function with a negative quadratic term would not be accurate enough for modeling haptic roughness perception.

Although the factors influencing tactile roughness perception are relatively well-studied, far less is known about the way the shape of the psychophysical roughness function is influenced by the interaction and combination of visual and haptic information. Lederman and Abbott (1981) reported comparable results in a visual and haptic roughness estimation experiment using abrasive paper. The way in which humans integrate visual and haptic texture information was shown to be well described by a weighted average model of both sources of sensory information in studies using matching and discrimination tasks as well as discrepancy paradigms (Jones \& O'Neil, 1985; Lederman \& Abbott, 1981; Lederman et al., 1986). These studies showed that both visual and haptic information are taken into account for bimodal texture perception, but that the relative bias between visual and haptic information can be altered depending on the texture dimension judged by the observer (e.g. roughness or spatial density). Moreover, orderings of natural stimuli based on physical characteristics (height variability) showed a similar correspondence with orderings based on visual or haptic perception (Bergmann Tiest \& Kappers, 2007). This indicates that both modalities are well suited for texture perception.

It is unclear to what extent the similarity of the two modalities can be explained by the subjects' level of familiarity with the stimulus material and the extensive training in its handling. If the visual stimulus is associated with a haptic memory, e.g., of the level of skin displacement and heat transfer into the material, the roughness percept arising for visual stimuli could be essentially haptic in nature. This would mean that different visual psychophysical curves could be expected for unfamiliar textures, e.g. textures used in a virtual reality setting providing only visual feedback. Alternatively, the visual sense could also be capable of directly judging roughness, for instance, by assessing the spatial density of a dot pattern texture. In that case, the system would need to resolve the different spatial resolutions of the haptic and the visual modality in case the roughness information from these two modalities is to be integrated. Studies investigating tactile acuity report threshold sizes between 1.21 and $3.50 \mathrm{~mm}$, with an age-related decline in tactile acuity of close to $1 \%$ a year, as summarized by Legge, Madison, Vaughn, Cheong, and Miller (2008). Average human visual acuity is better and corresponds to an image of about $0.005 \mathrm{~mm}$ on the retina (Sekuler \& Blake, 2001). A person with normal visual acuity (defined as the ability to resolve lines separated by one arcmin) would be able to resolve a spatial period of about $0.4 \mathrm{~mm}$ at a viewing distance of $75 \mathrm{~cm}$.

The present study aimed to characterize visually, haptically and visuo-haptically perceived roughness as a function of inter-dot spacing in a series of five experiments using unfamiliar dot patterns. The unfamiliarity with the stimulus material ensured that pre-existing differences in the haptic and visual experience with the stimulus material were negligible. In the first experiment we tested whether surface roughness is judged differently when subjects are provided with only visual or only haptic information, and how visual and haptic information are combined when both visual and haptic information are available. In addition, we studied whether roughness estimates are influenced by the modality in which the stimuli are presented first, i.e. by sensory familiarity with the stimulus material.

The visual stimuli in experiment 1 were computer-rendered images of models of the real textures to enable future use of this set-up in a magnetic resonance (MR) scanner with restricted viewing of the hand. In order to rule out the possibility that our findings resulted from unintended differences between real and virtual stimuli, we repeated the experiment with a direct visual presentation of the same textures used for the haptic modality condition. Similar findings in the first and the second experiment would indicate the suitability of the experimental design for neuroimaging studies, and would establish a more direct link between behavioral and neuroimaging results. In a third experiment we investigated whether visual and haptic roughness judgments are automatically combined or whether this changes in a unimodal task context that does not explicitly require the combination of visual and haptic information. In the fourth experiment we investigated whether the inherent difference in spatial resolution between vision and touch affects unimodal roughness judgments by extrapolating the stimulus set to include visual textures with finer spatial detail, i.e. close to and below the visual spatial acuity threshold. When stimuli are presented on a monitor without any scale reference (e.g. an image of the hand) as the case in experiment 4 , it is conceivable that a difference in estimated size of the visual textures with respect to the more natural haptic presentation condition might influence visual roughness perception. An over- or underestimation of the texture size could in turn result in a miscalculation of the size of the texture elements. In a study by Bergmann Tiest and Kappers (2007) subjects reported that next to the number and depth of indentations also the size of them influenced their visual roughness decisions of everyday materials. The fifth experiment was designed to account for the possibility that a difference in estimated size might have influenced visual roughness perception by presenting different scale references (i.e., different hand sizes) next to the stimuli for size comparisons.

We expected that either a linear or an inverted U-shape model would be suitable to capture the characteristics of the relationship of inter-dot spacing and roughness perception, in accordance with the two dominant findings in the literature mentioned above. For modeling the inverted U-shape function we used a bi-exponential model, which is characterized by a negative exponential with a steep slope followed by an exponential decrease with a shallower slope. An example of the asymmetric shape of such a bi-exponential function is presented in Fig. 1. This shape matches the asymmetric inverted U-shape profile of the data presented in previous studies 


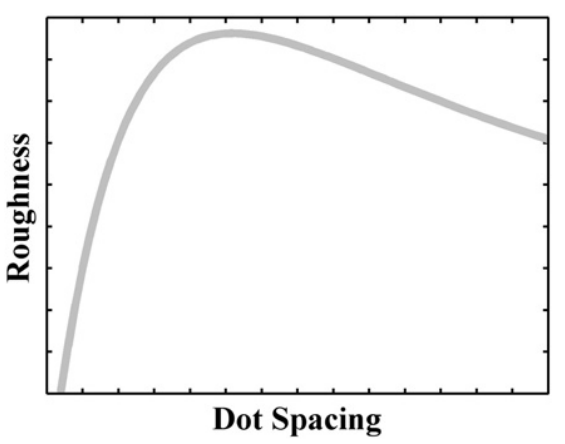

Fig. 1. Model of the bi-exponential function.

(Connor et al., 1990; Gescheider et al., 2005; Merabet et al., 2004, 2007). Other studies addressed the problem of modeling non-normal distributions by applying log-log transformations to the data (Klatzky \& Lederman, 1999; Lederman et al., 1999), which has the effect of normalizing positively skewed distributions and has the added benefit of variance stabilization. However, this approach would make it difficult to attain one of the main goals of the present study: a direct comparison of a linear model (in untransformed space) and a model capturing the inverted U-shape (in transformed space). In order to enable this comparison, we chose to use a relatively simple asymmetric function, the bi-exponential function, which can be used to fit the roughness as perceived by the subjects in the non-transformed space.

We compared the linear and the bi-exponential function for all subjects individually and separately for the visual, haptic and the bimodal condition. When taking the roughness judgment results for familiar textures (abrasive paper) by Lederman and Abbott (1981) into account, we would expect to find no significant difference between roughness perceived by touch and vision. However, we cannot be certain whether those results can be generalized to the kind of unfamiliar textures we used, because it was demonstrated that factors like task and stimulus material influence the effect of sensory modality on texture perception (Guest \& Spence, 2003a; Heller, 1989; Lederman et al., 1986). In the case that magnitude estimates are discrepant between the visual and haptic condition, we would expect to find a stronger emphasis on haptic than on visual information for bimodal roughness judgments in accordance with the results of a crossmodal discrepancy experiment conducted by Lederman et al. (1986).

\section{General method}

\subsection{Participants}

The study was approved by the local ethics committee. All participants were graduate and undergraduate students recruited via an advertisement at Maastricht University. Subjects gave written informed consent, were naïve to the hypotheses and received course credit or monetary compensation for the participation in the experiment. Participants had normal or corrected-to-normal vision and subjects with callused fingertips or a history of injury to the dominant hand were excluded.

\subsection{Stimuli and apparatus}

The stimuli consisted of $50 \times 50 \mathrm{~mm}$ plastic plates with different embossed dot patterns. The dots were $0.6 \mathrm{~mm}$ in elevation and $0.8 \mathrm{~mm}$ in diameter with smoothed edges to prevent any harm to the skin. The dots on the plates were arranged non-periodically. The only characteristic varying between the surfaces was the mean center-to-center dot spacing. The algorithm used to produce a different inter-element spacing was derived from a study by Lederman et al. (1986). First, a regular dot-matrix was produced with a specified inter-dot spacing. Afterwards, the dots were randomly jittered with a particular radius to create a non-regular pattern while preserving the average inter-dot spacing. This jitter radius ranged from $0.045 \mathrm{~mm}$ for dot patterns with the smallest spacing to $2.125 \mathrm{~mm}$ for textures with the biggest spacing. For each dot matrix a 3D wireframe model was created and computer-rendered in AutoCAD 2010 (Autodesk Inc., San Rafael, CA, USA) in order to create a set of matching visual stimuli. Two distant light sources following the direction of the viewpoint provided the lighting of each stimulus in such a way that all faces of the model were illuminated (see Fig. 2).

For the haptic stimulus presentation, the textures were arranged around the outside edge of a rotating circular wooden plate with a diameter of $52 \mathrm{~cm}$ and were covered with a second wooden plate. A rectangular cut-out in the cover plate enabled subjects to touch only one stimulus at a time. In addition, an opaque cloth curtain hid the stimulus from view. In all but one experiment (i.e., the second one) were the corresponding visual stimuli presented on a Full HD monitor centered in front of the subjects (see Fig. 3). The choice to present the textures on a monitor instead of direct visual exploration of the real stimuli was made since a similar study was planned inside a magnetic resonance imaging (MRI) scanner with restricted viewing conditions; presenting the textures on a monitor allows the comparison of data obtained inside and outside the scanner. Furthermore, it was previously shown that visual roughness estimation changes with variations in illumination (Ho, Landy, \& Maloney, 2006) and viewpoint (Ho, Maloney, \& Landy, 2007). The presentation of the textures on a screen enabled us to control those factors for all subjects and trials. A single lighting model and the frontoparallel viewpoint for all dot patterns provided constant depth information across all visual stimuli. The distance between the eyes of the subject and the monitor was kept constant between 70 and $80 \mathrm{~cm}$ and the stimuli subtended $5^{\circ}$ of visual angle.

\subsection{Procedure}

In experiments $1-3$, subjects sat at a table on which the stimulus presentation plate was placed along with a monitor behind it. In experiments 4 and 5 , the setup was the same excluding the stimulus presentation plate which was not needed in a mere visual task. Furthermore, a chin-rest was used in experiments 4 and 5 to provide an objective control for the distance between the subject's eyes and the monitor. Subjects were informed that they would be presented with one texture at a time and were asked to evaluate the roughness of this texture. No specific roughness definition was provided. The order of the stimulus presentation was randomized. Prior to the start of each condition, subjects were familiarized with the task and the stimuli in practice trials. During practice example textures with increasing inter-dot spacing were presented in ascending order. This procedure was used to enable subjects to choose an appropriate rating scale for each sensory modality (Dépeault et al., 2009; Meftah et al., 2000). In all experiments in which the visual stimuli were presented on a monitor, participants were instructed to focus on the screen at all times throughout the experiment. On every trial the exploration period was preceded by the presentation of a fixation cross on the screen. This time was used by the experimenter to rotate the presentation table to the correct stimulus. In the visual-haptic condition participants were instructed to inspect the haptic and the identical visual texture simultaneously. Since it was previously demonstrated that there is only a negligible accuracy difference between the preferred and non-preferred hand (Heller, 1982; Jones \& O'Neil, 1985), the dominant hand was used exclusively for all trials. Subjects were permitted to scan the textures with their index, middle and ring fingers without any time constraints, but were restricted to 


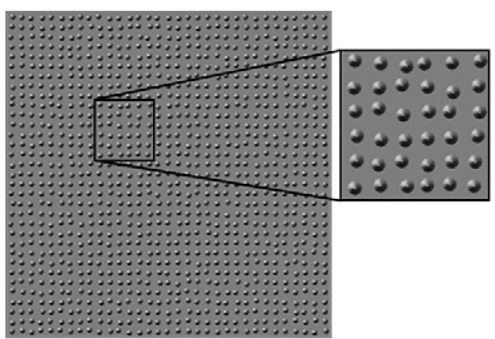

(a) $1.50 \mathrm{~mm}$

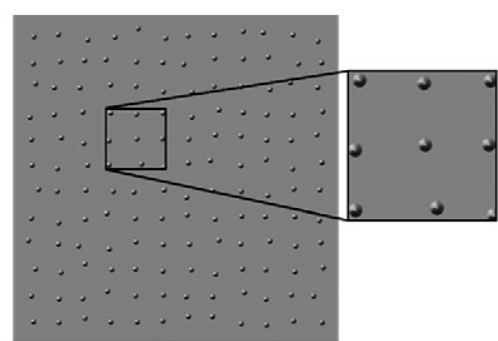

(b) $4.00 \mathrm{~mm}$

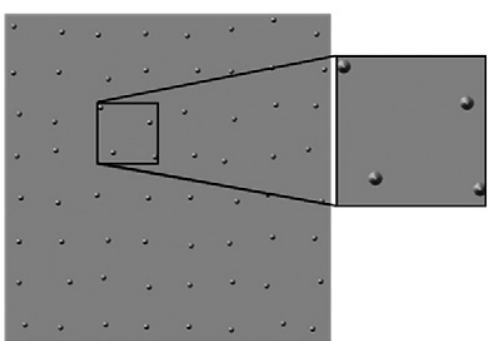

(c) $6.50 \mathrm{~mm}$

Fig. 2. Downscaled examples of the surfaces presented to the subjects.

exploration movements toward and away from the body. In addition, they were asked to keep the same contact force for all trials. The kind of haptic exploration was predetermined to minimize differences in ratings due to different exploration strategies between conditions and subjects. After subjects finished the examination of the stimulus they used a visual analogue scale (VAS) presented on the bottom of the screen to rate the roughness of the texture from very smooth to very rough by moving a red slider via the mouse with their non-dominant hand. The experimental procedure is summarized in Fig. 4.

\subsection{Data analysis}

The data were analyzed with SPSS 18 (SPSS Inc., Chicago, IL, USA). The width of the VAS for roughness scores was set to 800 pixels. The scores of all subjects were linearly transformed to a value range between 0 and 1 in order to simplify the interpretation of the results. The range of the scale was used for the transformation rather than the actual minimum and maximum ratings of the subjects in order to retain possible differences between subjects and modality conditions. These transformed values were used for all subsequent analyses. The level of significance was set to $\alpha=0.05$ for all analyses.

\section{Experiment 1}

The first experiment aimed at investigating roughness perception of unfamiliar dot pattern textures in three sensory modality conditions. Based on inconsistent findings in the literature (Connor et al., 1990; Dépeault et al., 2009; Gescheider et al., 2005; Meftah et al., 2000; Merabet et al., 2004, 2007), we addressed the question whether perceived surface roughness is better represented by a linear or bi-exponential function of inter-element spacing. Furthermore, as

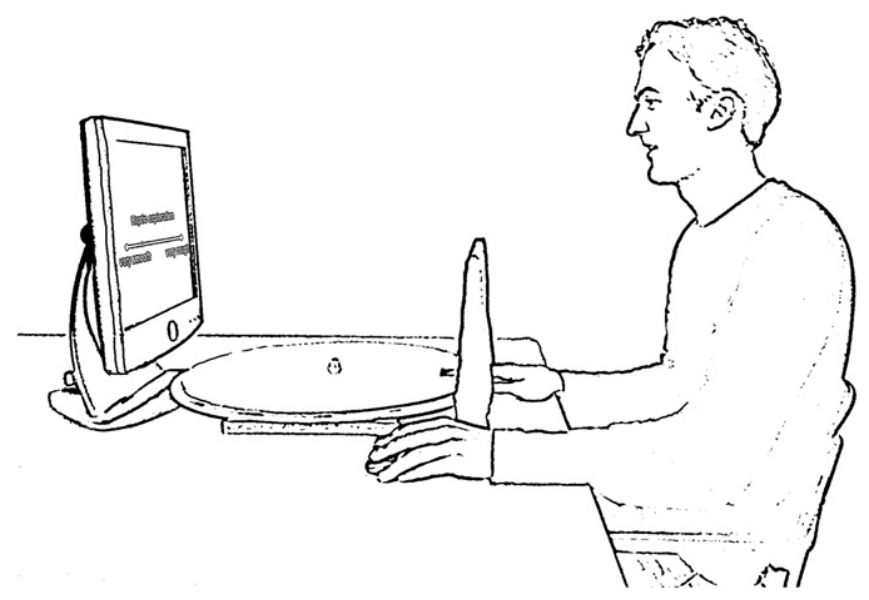

Fig. 3. Schematic representation of the experimental setup. previous studies showed that visual and haptic information might be weighted differently in texture perception (Guest \& Spence, 2003a; Heller, 1989; Lederman et al., 1986), we asked whether roughness perception changes with the kind of sensory information available as well as with the first sensory experience with the stimulus material.

\subsection{Method}

\subsubsection{Participants}

Thirty subjects, 18 women and 12 men, mean age 28.5 years, participated in this experiment. Twenty-six of the participants were right-handed.

\subsubsection{Stimuli and apparatus}

In this experiment 29 textures were presented. The mean center-to-center dot spacing of the stimuli ranged from 1.50 to $8.50 \mathrm{~mm}$ and increased in steps of $0.25 \mathrm{~mm}$.

\subsubsection{Procedure}

The roughness estimation task was performed for three different modality conditions: visual, haptic and a combined visual-haptic condition. The order of conditions was counter-balanced across subjects and all textures were presented three times in each condition. Since the 29 stimuli could not be arranged on a single plate due to practical limitations with respect to the diameter of the plate, 15 stimuli were placed on one presentation plate and the other 14 on a second one. The assignment of the 29 stimuli to the plates was random and changed for each subject. During practice textures with an inter-dot spacing of $1.50,3.25,5.00,6.75$ and $8.50 \mathrm{~mm}$ were presented once.

\subsubsection{Data analysis}

One aim of this study was to reveal the direction of the sensory information bias toward haptic or visual information for the bimodal roughness estimates. To that end a mixed-design analysis of variance (ANOVA) with the within-subject factors modality (3) and inter-dot spacing (29) and the between-subject factor first modality condition (3) was employed for the roughness scores. The Greenhouse-Geisser correction was applied whenever sphericity could not be assumed.

Furthermore, a roughness function for each subject and sensory modality was modeled by fitting a linear and bi-exponential function to the data collapsed over all repetitions. The bi-exponential model was represented by the following equation:

$r(d)=\beta_{0}+e^{\left(-\beta_{2}\left(d-\beta_{1}\right)\right)}-e^{\left(-\beta_{3}\left(d-\beta_{1}\right)\right)}$

where $r(d)$ is the roughness score as a bi-exponential function of inter-dot distance and $\boldsymbol{\beta 0}$ to $\boldsymbol{\beta 3}$ are constant terms. The first two constants $\beta \mathbf{0}$ and $\boldsymbol{\beta 1}$ model the vertical and horizontal offset of the function while $\beta 2$ and $\beta 3$ model the shape of both exponentials. The benefit of this function over a negative quadratic function is the possibility to model asymmetric profiles of the psychophysical roughness 


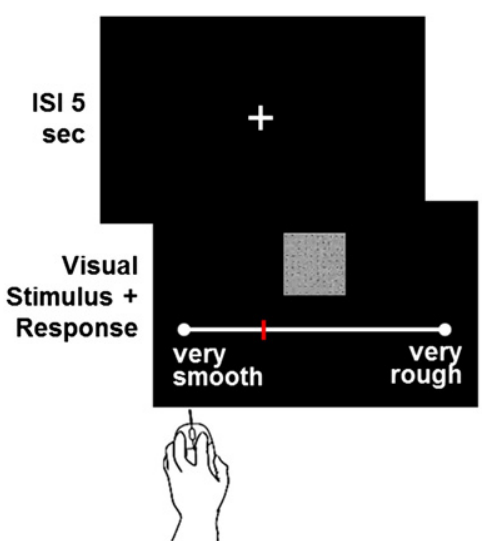

(a) Visual condition

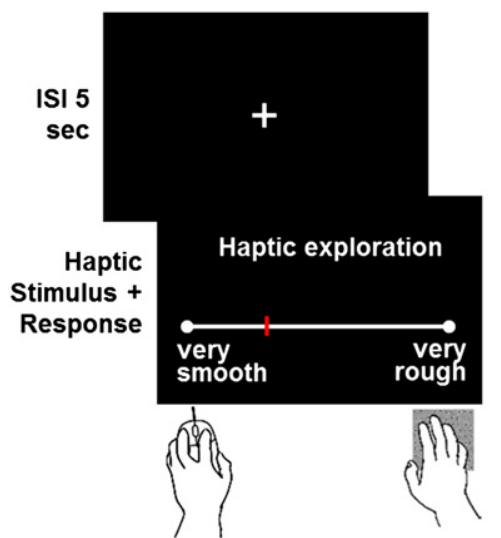

(b) Haptic condition

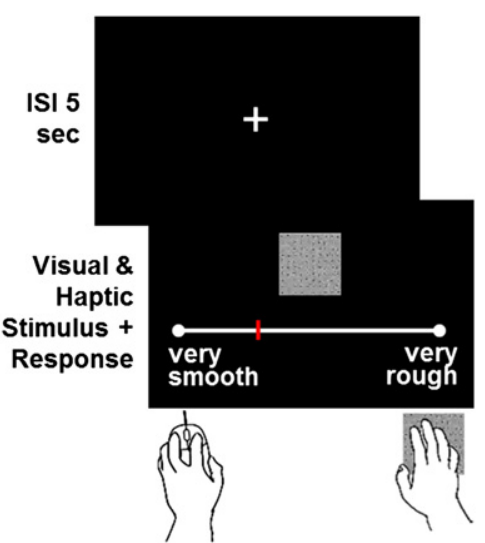

(c) Visual-Haptic condition

Fig. 4. Experimental paradigm. ISI = inter-stimulus interval.

function as explained in the introduction. Both models were compared for each condition and subject with the corrected version of Akaike Information Criterion (AICc). The AIC is a model selection method that identifies the best descriptive model for the data within a set of a priori considered models. This method was chosen because it can be applied to the comparison of non-nested models (Burnham (a) Average Across All First-Condition Groups

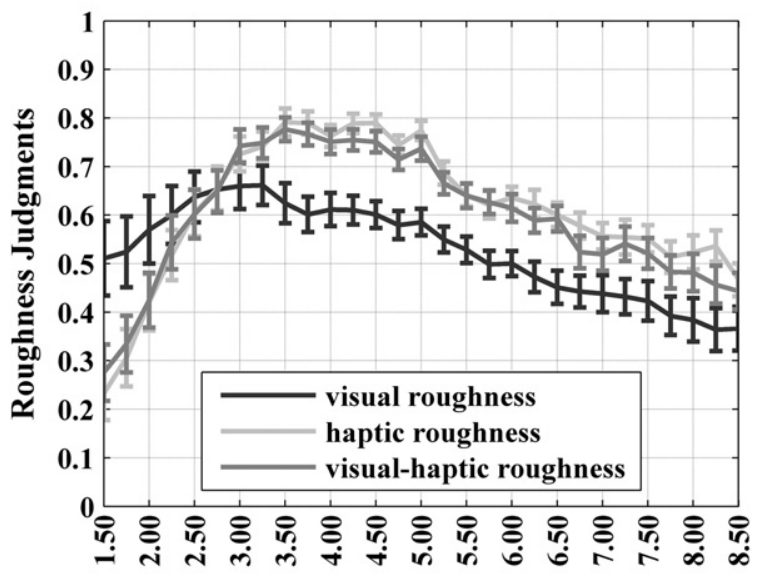

Dot Spacing in $\mathbf{m m}$

(c) Haptic Roughness

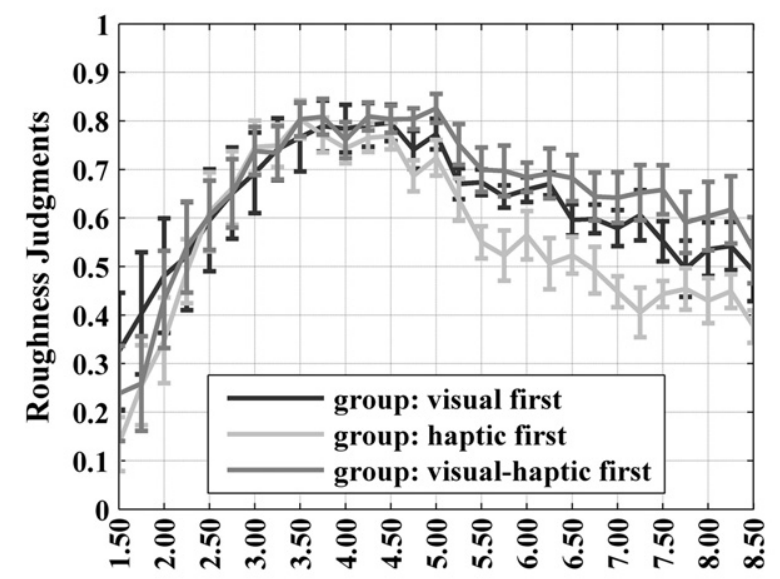

Dot Spacing in $\mathbf{m m}$ (b) Visual Roughness

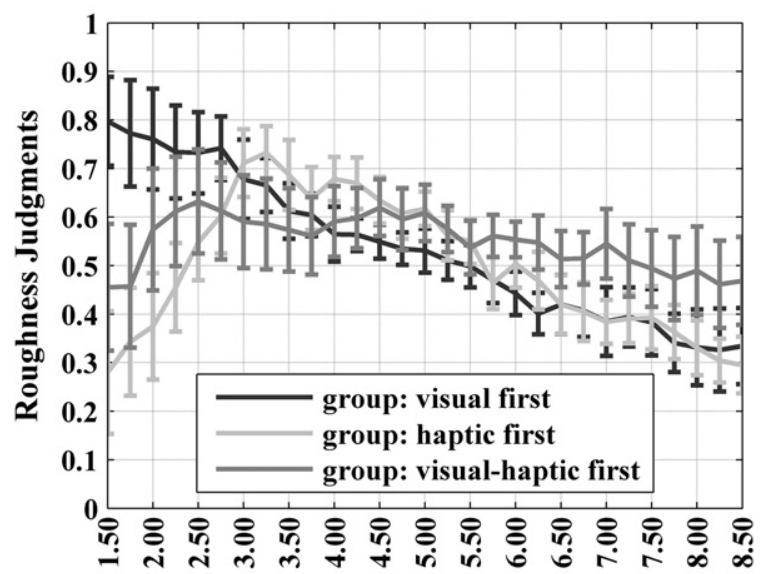

Dot Spacing in $\mathbf{m m}$

(d) Visual-Haptic Roughness

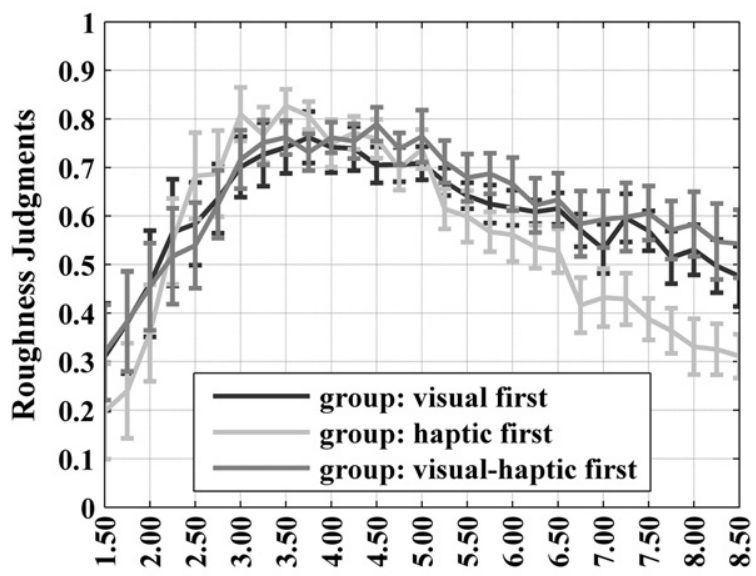

Dot Spacing in $\mathbf{m m}$

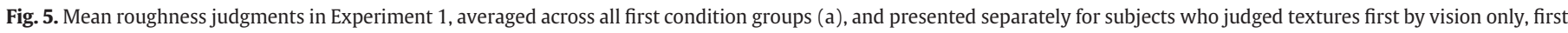

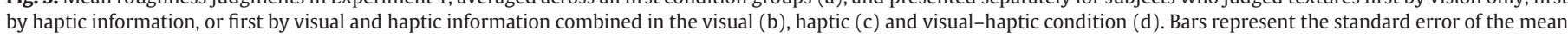
(SEM). 
\& Anderson, 2004; Wagenmakers \& Farrell, 2004) and takes the number of included parameters into account. Furthermore, a small-sample corrected version of this criterion is available (AICc) and was used for the present data as the number of data points divided by the number of free parameters is smaller than 40 , which was previously recommended as the approximated minimum value for using the uncorrected version (Burnham \& Anderson, 2004). Akaike weights $\left(w_{i}\right)$, representing the relative probability that model $\mathrm{i}$ is the best model for the data under consideration of the set of models, were used to decide in favor of the bi-exponential model, when the probability was above $50 \%$.

\subsection{Results}

The graphs in Fig. 5(a) suggest that roughness judged by vision is on average attenuated in comparison to the haptic and visual-haptic judgments. This influence of modality on roughness estimation was supported by a significant main effect of the within-subject factor modality in the mixed-design ANOVA of the roughness scores, $F(1.72,46.37)=12.45, p<0.001$, partial $\eta^{2}=0.32$. Post hoc paired t-tests confirmed that textures were judged significantly less rough in the visual condition than in the haptic condition, mean difference: $-0.09, p<0.001$, and compared to the bimodal condition, mean difference: $-0.07, p<0.01$. This was not true for the direct comparison of the haptic and bimodal condition, mean difference: $0.02, p=0.83$.

Furthermore, the main effect of the within-subject factor inter-dot spacing, $F(1.79,48.42)=15.99, p<0.001$, partial $\eta^{2}=0.37$, and the Modality $\times$ Inter-Dot Spacing interaction, $F(5.21,140.71)=10.26$, $p<0.001$, partial $\eta^{2}=0.28$ were significant. This two-way interaction is also implied by Fig. 5(a) showing that visual roughness exceeds roughness perceived by touch and bimodally for small inter-dot spacings while this effect is reversed for bigger dot distances. Based on the literature showing that perceived roughness peaks at about $3 \mathrm{~mm}$ inter-dot spacing, we used this cut off point to pool roughness judgments $\leq 3 \mathrm{~mm}$ inter-dot spacing and data acquired for textures $>3 \mathrm{~mm}$ inter-dot spacing into two groups. In post hoc contrasts all modality conditions were compared for the group of small and big inter-dot distances in order to describe the two-way interaction further. These tests confirmed partially what is indicated by Fig. 5(a), namely significantly higher visual roughness scores for smaller inter-dot spacings, mean difference between the visual and haptic condition: $0.69, p<0.05$, but attenuated visual roughness scores for bigger inter-dot spacings, mean difference between the visual and haptic condition: $-3.15, p<0.001$ and mean difference between the visual and bimodal condition: $-2.58, p<0.001$; all other comparisons were not significant.

The mixed-design ANOVA also showed a significant three-way interaction of Modality $\times$ Inter-Dot Spacing $\times$ First Condition, $F(10.42$, $140.71)=2.15, p<0.05$, partial $\eta^{2}=0.14$. Fig. 5(b) suggest that this interaction is caused by a difference in visually perceived roughness between subjects who experienced the textures first by vision as compared to all other subjects, but for small inter-dot spacings only. Fig. 5(c)-(d) indicates further that subjects who evaluated the textures first in the haptic condition show attenuated haptic and bimodal roughness scores as compared to both other first condition groups. In order to test the cause of this interaction we pooled the data into the same small and big dot distance groups as described above and compared in post-hoc contrasts the roughness estimates between all first condition groups separately for all modality conditions. These post hoc tests confirmed for small inter-dot spacings a significant difference in estimated visual roughness between subjects who experienced the textures first in the visual and first in the haptic condition, mean difference: $1.90, p<0.05$. Haptic roughness judgments of bigger inter-dot spacings were on average attenuated when subjects experienced the textures first by touch, mean difference between haptic and visual first condition group: $-1.68, p<0.05$ and mean difference between haptic and visual-haptic first condition group: $-2.68, p<0.005$. All other post hoc contrasts did not reach significance.

Table 1 summarizes the results of fitting a linear and bi-exponential model to the data of each subject and condition. In total, 12 out of 30 subjects showed a linear relationship between visual roughness estimates and inter-dot spacing of the textures. Five out of those subjects evaluated the textures first by vision, three first by touch and four first in the visual-haptic condition. In contrast, in the haptic condition only three out of the entire sample of 30 subjects showed a linear relationship between inter-dot spacing and roughness estimates (two subjects with the visual condition first and one with the bimodal condition first). This was also true for five subjects in the visual-haptic condition, two of whom were first confronted with the visual condition, two with the haptic condition and one with the bimodal condition.

\subsection{Discussion}

Previous research failed to show a difference between visual and haptic roughness estimation of abrasive paper (Lederman \& Abbott, 1981). The results of the present study extend those earlier findings for a set of unfamiliar textural stimuli by showing that roughness estimates were on average slightly attenuated when only visual information of the textures was available.

Haptic and bimodal roughness perception of unfamiliar textural stimuli appears to be best described by a bi-exponential function, with a steep rise up to an inter-dot spacing of about 3-4 $\mathrm{mm}$ and a slighter decrease for textures with bigger dot spacing. Klatzky \& Lederman, 1999 found a similar increase in perceived roughness for haptic texture exploration up to $3.50 \mathrm{~mm}$ dot spacing with the bare finger. Despite the close resemblance of the findings for closely spaced dot patterns, there are several noteworthy differences in the design and analysis of Klatzky and Lederman's (1999) study. First, their maximum inter-element distance in the stimulus pool was $3.50 \mathrm{~mm}$. Therefore, they could only estimate a theoretical peak based on the fitted function, which was about $4.8 \mathrm{~mm}$ for the bare finger. However, this theoretical peak might differ from empirical values. By extending the stimulus range to textures with bigger inter-dot separations, other studies empirically determined the peak to be between 3 and $4 \mathrm{~mm}$ (Connor et al., 1990; Gescheider et al., 2005; Merabet et al., 2007), which is consistent with our haptic results. Furthermore, Klatzky and Lederman transformed their data to $\log -\log$ space before applying a quadratic function. The fit of a quadratic function to log-log transformed roughness data of dot patterns should be comparable to the fit of the bi-exponential function to only linearly transformed data in the present study. We decided to use only linear transformations on our data, because we wanted to test against a linear model and because data presentation in plots with linear scales is more intuitive. Our results indicate that the same inverted U-shape function can be generalized to bimodal visualhaptic roughness perception of unfamiliar dot pattern textures. In contrast to the haptic and bimodal condition, visual roughness perception showed a trend for a more linear relationship with respect to inter-element spacing of textures, as evidenced by the model fitting results as well as the significant interaction of modality and dot distance in the ANOVA.

The different results for the visual modality might be explained by a difference in spatial resolution. It is known that fine spatial detail is better resolved by the eye than by the skin (Lederman \& Klatzky, 2009). When touching textures with very small inter-dot spacing the finger pads might not be able to resolve the single elements due to the restricted compliance of the skin resulting in a relatively smooth texture percept. For the same small inter-dot spacing, the eye would be expected to resolve the single elements. Hence, the textures might have appeared rough to the eye because of the big number of dots and the small spacing between them. With increasing 
Table 1

Summary of the model comparisons of the linear and bi-exponential model for all subjects and conditions (Experiment 1).

\begin{tabular}{|c|c|c|c|c|c|c|c|c|c|}
\hline & \multirow{2}{*}{$\begin{array}{l}\text { First } \\
\text { condition }\end{array}$} & \multicolumn{4}{|c|}{ Linear model selected } & \multicolumn{4}{|c|}{ Bi-exponential model selected } \\
\hline & & $\begin{array}{l}\text { No. of } \\
\text { subjects }^{\text {a }}\end{array}$ & $\begin{array}{l}\text { Range of } \mathrm{R}^{2} \\
\text { linear }^{\mathrm{b}}\end{array}$ & $\begin{array}{l}\text { Range of } \mathrm{R}^{2} \\
\text { bi-exponential }^{\mathrm{b}}\end{array}$ & $\begin{array}{l}\text { Range of } \mathrm{w}_{\mathrm{i}}(\mathrm{AICC}) \\
\text { bi-exponential }\end{array}$ & $\begin{array}{l}\text { No. of } \\
\text { subjects }^{\text {a }}\end{array}$ & $\begin{array}{l}\text { Range of } \mathrm{R}^{2} \\
\text { linear }^{\mathrm{b}}\end{array}$ & $\begin{array}{l}\text { Range of } \mathrm{R}^{2} \\
\text { bi-exponential }^{\mathrm{b}}\end{array}$ & $\begin{array}{l}\text { Range of } \mathrm{w}_{\mathrm{i}}(\mathrm{AICc}) \\
\text { bi-exponential }\end{array}$ \\
\hline \multirow[t]{3}{*}{ Visual condition } & $\mathrm{v}$ & 5 & $0.81-0.96$ & $0.82-0.97$ & $0-32 \%$ & 5 & $0.85-0.96$ & $0.96-0.98$ & $98-100 \%$ \\
\hline & $\mathrm{h}$ & 3 & $0.90-0.95$ & $0.90-0.94$ & $1-32 \%$ & 7 & $0.02-0.43$ & $0.54-0.85$ & $88-100 \%$ \\
\hline & vh & 4 & $0.92-0.97$ & $0.89-0.96$ & $0-31 \%$ & 6 & $0.15-0.92$ & $0.81-0.95$ & $84-100 \%$ \\
\hline \multirow[t]{3}{*}{ Haptic condition } & $\mathrm{v}$ & 2 & $0.89-0.93$ & $0.83-0.88$ & $0 \%$ & 8 & $0.04-0.97$ & $0.66-0.99$ & $100 \%$ \\
\hline & $\mathrm{h}$ & 0 & - & - & - & 10 & $0.00-0.69$ & $0.70-0.90$ & $100 \%$ \\
\hline & $\mathrm{vh}$ & 1 & 0.89 & 0.89 & $7 \%$ & 9 & $0.00-0.72$ & $0.75-0.93$ & $100 \%$ \\
\hline \multirow[t]{3}{*}{ Visual-haptic condition } & $\mathrm{v}$ & 2 & $0.77-0.89$ & $0.80-0.89$ & $4-25 \%$ & 8 & $0.06-0.95$ & $0.78-0.97$ & $100 \%$ \\
\hline & $\mathrm{h}$ & 2 & $0.76-0.89$ & $0.78-0.86$ & $0-15 \%$ & 8 & $0.00-0.35$ & $0.69-0.90$ & $100 \%$ \\
\hline & $\mathrm{vh}$ & 1 & 0.94 & 0.94 & $12 \%$ & 9 & $0.00-0.66$ & $0.56-0.91$ & $100 \%$ \\
\hline
\end{tabular}

Note. Model decision was based on $\mathrm{w}_{\mathrm{i}}(\mathrm{AICc}) . \mathrm{v}=$ visual, $\mathrm{h}=$ haptic, $\mathrm{vh}=$ visual-haptic.

a There is a total of 10 subjects in each first condition group.

b $\mathrm{R}^{2}$ coefficient of determination.

inter-dot distance, the density of elements on the surface decreases which resulted in a linear decrease in visually perceived roughness. If this line of reasoning applies, we would expect to find a linear relationship between inter-dot spacing and roughness perception for all subjects in the visual condition. However, the trend for a linear relationship between inter-element spacing and visual roughness estimations is weaker for subjects who experienced the stimuli first in a condition with haptic input, which is also represented by a significant interaction of modality, spacing and first condition in the ANOVA. This might indicate that subjects, who had a haptic anchor point to rely on, provided by previous haptic experience with the textures in the haptic and/or bimodal condition, tended to imagine how the visually presented textures would feel when being touched. In the remaining text, we shall refer to this imagery of the somatosensory experience related to the act of touching the textures as haptic imagery, which could be interpreted as a calibration of visual roughness judgments to haptic roughness perception. Such a calibration would be in line with the general notion of people that roughness is better evaluated by touch than by vision as, for example, indicated in a small survey with ten individuals by Lederman et al. (1986). This application of a lower resolution haptic filter on the visual roughness estimates is particularly beneficial when individuals are asked to combine both visual and haptic information in a single bimodal roughness rating. Choosing a haptic imagery strategy promotes the development of a consistent roughness concept between modalities in favor of multisensory judgments. During debriefing some of our subjects indeed reported that they used haptic imagery in the visual condition.

The general superiority of the bi-exponential model in the haptic and bimodal condition vs. the increased incidence of the linear model in the visual condition supports the findings from previous studies (Heller, 1982; Lederman et al., 1986), showing that visualhaptic roughness judgments of textures are in general more influenced by haptic than by visual information. This is further confirmed by our finding that the difference between bimodal and unimodal haptic roughness estimates is negligible, while this is not true for the comparison of visual and bimodal roughness judgments. The consistency of our results with previous findings indicates that the haptic percept receives a stronger weight, possibly because it incorporates information about the interaction of the texture and skin which might be relevant for object manipulation.

\section{Experiment 2}

The second experiment was designed to test whether the results of the first experiment would generalize to natural viewing conditions. In the first experiment visual textures were presented on a monitor. Despite the fact that the visual stimuli were designed to be as similar as possible to the real textures, it is conceivable that unintended differences between the haptic and visual stimulus material influenced the results of experiment 1 . Furthermore, it is known that the integration of multisensory information is optimal when the information is presented in a temporarily synchronous and spatially coincidental way, indicating that the information comes from a common source (Helbig \& Ernst, 2007a,b). In the first experiment subjects were told that the stimuli presented on the screen were the same stimuli they touched. Nevertheless, the discrepant location of the visual and haptic information might have influenced cue integration across sensory modalities. Furthermore, during the exploration of the textures the hand of the subject was hidden by a cloth curtain in all three modality conditions of experiment 1 . However, Heller (1982) suggested that the visibility of the hand can influence bimodal performance. He argued that visual guidance of tactual exploration has a positive effect on bimodal texture discrimination, improving bimodal versus unimodal performance in his study. In order to account for the possibility that results of experiment 1 were influenced by the virtual presentation of the textures on a monitor, the same experiment was repeated with a more natural viewing condition.

\subsection{Method}

\subsubsection{Participants}

Eighteen volunteers, all right handed, seven males and 11 females, mean age 23.2 years, participated in this study.

\subsubsection{Stimuli and apparatus}

The same stimulus material and apparatus as described in experiment 1 were used. The experiment was conducted in a room without windows and with fluorescent lamps on the ceiling. All lights were switched on so that the room was evenly lit, equaling the lighting conditions in a normal office environment.

\subsubsection{Procedure}

Subjects performed the same roughness estimation task as described in experiment 1 . In experiment 2 , however, the same real textures were used for the haptic, visual and bimodal condition, all presented on a wooden circular board. Furthermore, subjects were able to see their own hand in the bimodal condition.

\subsubsection{Data analysis}

The same analyses as in experiment 1 were performed. These included a mixed-design ANOVA with the within-subject factors modality (3) and inter-dot spacing (29) and the between-subject factor first modality condition (3) as well as the model fitting of the linear and bi-exponential function. When sphericity was violated the Greenhouse Geisser adjustment for degrees of freedom was used in the ANOVA. 


\subsection{Results}

The mixed-design ANOVA showed a significant main effect of the within-subject factor inter-dot spacing, $F(1.67,25.02)=23.95$, $p<0.001$, partial $\eta^{2}=0.62$. However, the main effects of the within-subject factor modality and the between-subject factor first modality condition did not reach significance, $F(1.41,21.17)=0.32$, $p=0.65$, partial $\eta^{2}=0.02$ and $F(2,15)=0.63, p=0.55$, partial $\eta^{2}=$ 0.08 , respectively.

The plot in Fig. 6(a) implies that visually perceived roughness of textures with small inter-dot spacings is on average higher as compared to haptic and visual-haptic roughness perception, while the opposite seems to be true for inter-dot spacings above $3 \mathrm{~mm}$. This was confirmed by a significant two-way interaction of Modality $\times$ Inter-Dot Spacing, $F(6.58,98.65)=11.59, p<0.001$, partial $\eta^{2}=0.44$. Post-hoc contrasts comparing all modality conditions for the pooled data of textures $\leq 3 \mathrm{~mm}$ and for roughness estimates $>3 \mathrm{~mm}$ showed similar effects as in experiment 1 , namely significantly higher visual judgments in the group of small inter-dot spacings, mean difference between the visual and haptic condition: 1.29, $p<0.001$ and mean difference between the visual and bimodal condition: 1.13, $p<0.001$. The effect was reversed for roughness judgments in the group of textures with big inter-dot spacing, mean difference between the visual and haptic condition: $-1.21, p<0.05$ and mean difference between the visual and bimodal condition: $-1.35, p<0.005$. The mixed-design ANOVA revealed also a significant interaction of the within-subject factor sensory modality and the between-subject factor first modality condition, $F(2.82,21.17)=6.25, p<0.005$, partial $\eta^{2}=0.46$, with haptic roughness scores being on average lower than bimodal roughness judgments when textures were judged first in the haptic condition, mean difference: $-0.07, p<0.005$, and higher when textures were first experienced in the bimodal condition, mean difference: 0.05 , $p<0.05$. The two-way interaction of First Condition $\times$ Inter-Dot Spacing did not reach significance in the mixed-design ANOVA, $F(3.34$, 25.02) $=1.22, p=0.32$, partial $\eta^{2}=0.14$.

Furthermore, there was a significant three-way interaction of Modality $\times$ First Condition $\times$ Inter-Dot Spacing, $F(13.15,98.65)=$ 1.91, $p<0.05$, partial $\eta^{2}=0.20$. The plot in Fig. $6(\mathrm{~b})$ suggests that this interaction is caused by a difference in visually perceived roughness of textures with inter-dot spacings $\leq 2.50 \mathrm{~mm}$ depending on the modality with which subjects experienced the textures first. Additionally, there seems to be an effect of the factor first condition on haptic roughness perception for textures with intermediate interdot spacing (2.75-5.00 mm) as presented in Fig. 6(c). We investigated these hypotheses by pooling the roughness judgments into three different groups of inter-dot spacing, i.e. $1.50-2.50 \mathrm{~mm}, 2.75-$ (a) Average Across All First-Condition Groups

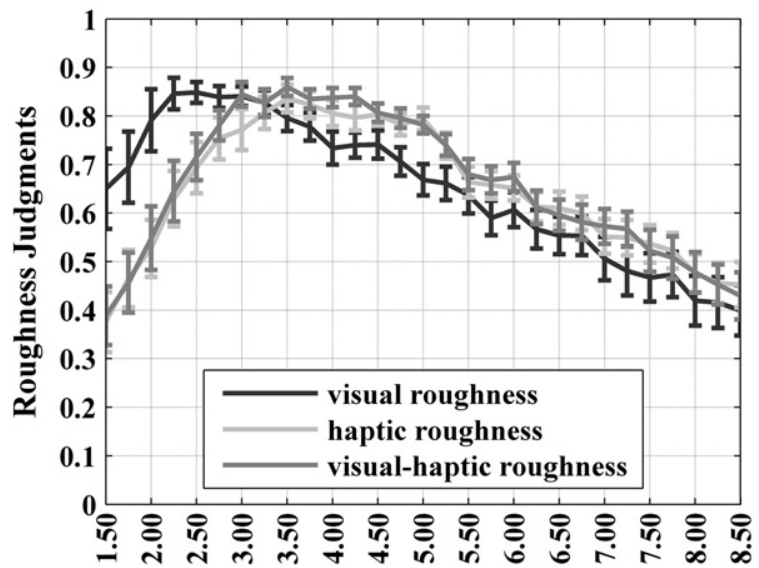

Dot Spacing in $\mathbf{m m}$

(c) Haptic Roughness

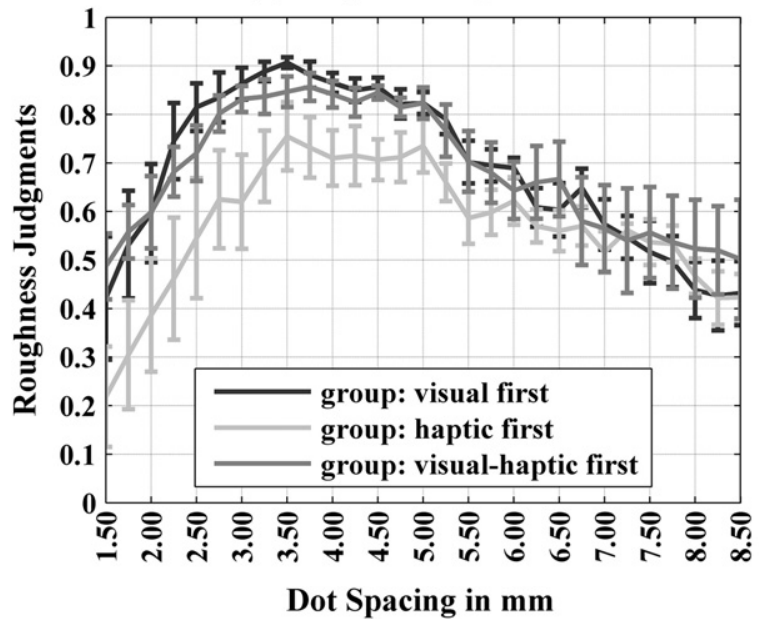

(b) Visual Roughness

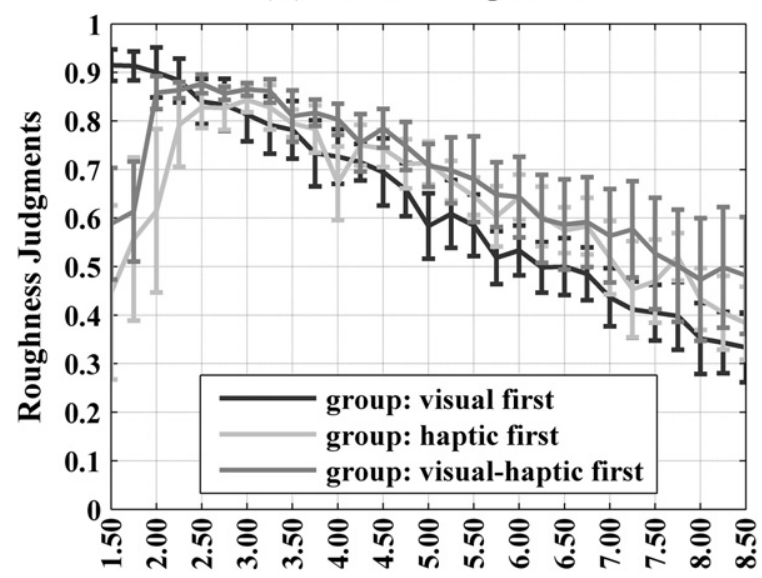

Dot Spacing in $\mathbf{m m}$

(d) Visual-Haptic Roughness

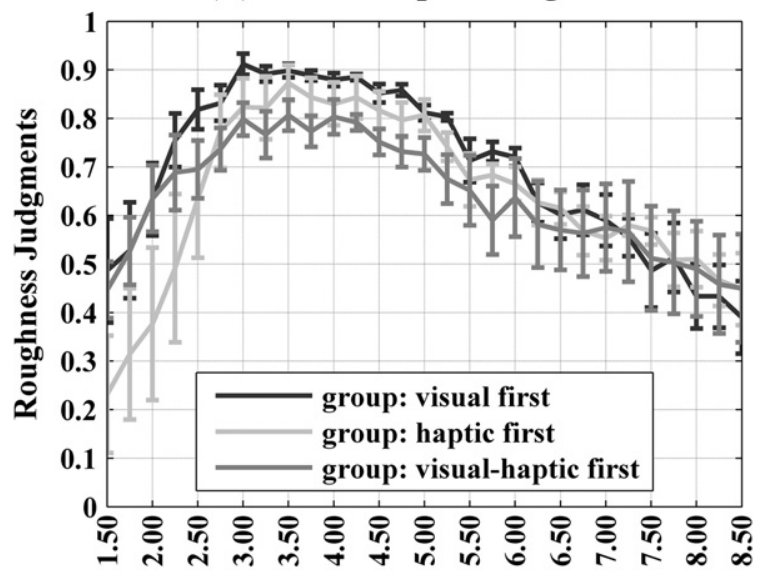

Dot Spacing in $\mathbf{m m}$

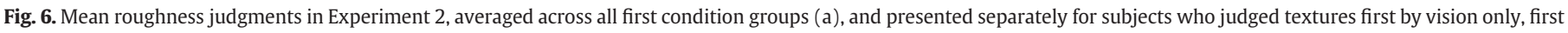

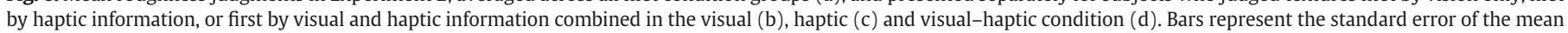
(SEM). 
$5.00 \mathrm{~mm}, 5.25 \mathrm{~mm}-8.50 \mathrm{~mm}$, and by comparing all first conditions within these groups separately for the different sensory modalities. The post-hoc contrasts confirmed significantly higher visual roughness estimates for inter-dot spacings $\leq 2.50 \mathrm{~mm}$ when subjects experienced the textures first in the visual as compared to the haptic condition, mean difference: $1.21, p<0.05$. In the haptic condition roughness judgments were attenuated for textures with intermediate inter-dot spacings when subjects experienced the textures first by touch, mean difference between the haptic and visual first condition group: $-1.58, p<0.01$ and mean difference between the haptic and bimodal first condition group: $-1.32, p<0.05$. There was also a significant effect of first condition on bimodal roughness judgments of textures with intermediate dot distances, i.e. with attenuated scores for subjects who experienced the textures first in the bimodal as compared to the visual condition, mean difference: $-1.02, p<0.05$.

The model fitting results are comparable to the findings of experiment 1 in which the visual textures were presented on a monitor. Only two out of all 18 subjects showed a better fit for the linear model in the haptic condition, with one having experienced the textures first in the visual condition and one in the visual-haptic condition. The same two subjects also showed a better fit for the linear model in the visual-haptic condition, while the bi-exponential model was superior for all other subjects. Only in the visual condition the incidence of the linear model increased with seven out of 18 subjects. In line with the results of experiment 1 , the highest incidence of the linear model can be found in the group of subjects who experienced the textures first by vision, i.e. four out of six participants, while only one subject who experienced the textures first by touch and only two subjects in the visual-haptic first condition group showed a better fit for the linear model (see Table 2).

\subsection{Discussion}

The findings of the second experiment using natural visual textures mainly confirm the results of the first experiment in which the visual stimuli were presented on a monitor. Haptic and bimodal roughness perception were well described by a bi-exponential function of inter-dot spacing peaking at about 3-4 mm, whereas the incidence of the linear function increased for visual roughness perception, as also indicated by the interaction of modality and inter-dot spacing in the ANOVA. This might be caused by a different spatial resolving power of the visual and haptic system, as already discussed in experiment 1 . This distribution difference of the linear and bi-exponential model for visual and haptic roughness perception supports our assumption of an influence of the spatial resolution of both senses on roughness perception of dot pattern textures. Again, there seems to be an effect of the first sensory experience with the stimulus material on visual roughness perception: there is a stronger tendency toward the bi-exponential model for visual roughness perception when subjects can refer to a haptic anchor point obtained from previous texture exploration in the haptic or bimodal condition. This effect of the first modality condition on visual roughness judgments was confirmed by the significant interaction of first condition, modality and inter-element spacing. Hence, our interpretation that individuals tend to calibrate their visual roughness estimates to haptic roughness perception when provided with haptic experience is further supported.

However, the results of the second experiment did not confirm the main effect of modality on roughness perception. We cannot rule out that this difference between the first and second experiments is caused by the different presentation modes of the visual stimuli, but the significant interaction of modality and spacing in both experiments indicates a similar trend. One possible explanation for the non-significant main effect of modality might be the relatively small effect of attenuated visual roughness scores for textures with inter-dot spacings above $3 \mathrm{~mm}$. This effect might have been cancelled out by the rather strong effect of increased visual roughness judgments for textures with smaller dot distances.

In general, the similarity of findings in experiments 1 and 2 leads to the conclusion that the presentation mode of visual stimuli has no or only a negligible influence on visual and bimodal roughness perception of dot pattern stimuli. Hence, the additional visibility of the exploring hand in the bimodal condition, as compared to the first experiment, did not change the overall results. This is in contrast to the findings of Heller (1982) who showed that the visibility of the exploring hand and not the visual texture information per se was crucial for a performance improvement in a bimodal as compared to a haptic texture discrimination task. He also reported that subjects showed a preference for haptic texture cues when visual information was available. This stronger weight of haptic information for bimodal judgments was confirmed in the present study for both virtual and natural visual presentation conditions. We did not find a change in bimodal perception between the virtual and the natural setting possibly because of the nature of the experimental task. Visual guidance of hand movements might be particularly beneficial when fine discriminations between textures are required, as in Heller's experiment for abrasive papers. Such fine relative judgments were not required from the participants in our experiment; hence no added value by visual guidance of the hand movements and therefore no change in the bimodal results are expected.

The similarity of findings in experiments 1 and 2 opens the possibility to translate the behavioral experimental design to virtual environments or to a setup with restricted viewing conditions, i.e. for future comparative neuroimaging studies. Such an approach might broaden our understanding of the neural correlates of visual-haptic bimodal roughness perception.

Table 2

Summary of the model comparisons of the linear and bi-exponential model for all subjects and conditions (Experiment 2).

\begin{tabular}{|c|c|c|c|c|c|c|c|c|c|}
\hline & \multirow{2}{*}{$\begin{array}{l}\text { First } \\
\text { condition }\end{array}$} & \multicolumn{4}{|c|}{ Linear model selected } & \multicolumn{4}{|c|}{ Bi-exponential model selected } \\
\hline & & $\begin{array}{l}\text { No. of } \\
\text { subjects }^{a}\end{array}$ & $\begin{array}{l}\text { Range of } \mathrm{R}^{2} \\
\text { linear }^{\mathrm{b}}\end{array}$ & $\begin{array}{l}\text { Range of } \mathrm{R}^{2} \\
\text { bi-exponential }^{\mathrm{b}}\end{array}$ & $\begin{array}{l}\text { Range of } \mathrm{w}_{\mathrm{i}}(\mathrm{AICc}) \\
\text { bi-exponential }\end{array}$ & $\begin{array}{l}\text { No. of } \\
\text { subjects }^{a}\end{array}$ & $\begin{array}{l}\text { Range of } \mathrm{R}^{2} \\
\text { linear }^{\mathrm{b}}\end{array}$ & $\begin{array}{l}\text { Range of } \mathrm{R}^{2} \\
\text { bi-exponential }^{\mathrm{b}}\end{array}$ & $\begin{array}{l}\text { Range of } \mathrm{w}_{\mathrm{i}}(\mathrm{AICc}) \\
\text { bi-exponential }\end{array}$ \\
\hline \multirow[t]{3}{*}{ Visual condition } & $\mathrm{v}$ & 4 & $0.88-0.94$ & $0.88-0.90$ & $0-23 \%$ & 2 & $0.92-0.95$ & 0.97 & $99-100 \%$ \\
\hline & $\mathrm{h}$ & 1 & 0.95 & 0.88 & $0 \%$ & 5 & $0.08-0.95$ & $0.68-0.95$ & $77-100 \%$ \\
\hline & vh & 2 & $0.92-0.95$ & $0.85-0.88$ & $0 \%$ & 4 & $0.01-0.28$ & $0.82-0.93$ & $100 \%$ \\
\hline \multirow[t]{3}{*}{ Haptic condition } & $\mathrm{v}$ & 1 & 0.94 & 0.92 & $0 \%$ & 5 & $0.01-0.43$ & $0.78-0.91$ & $100 \%$ \\
\hline & $\mathrm{h}$ & 0 & - & - & - & 6 & $0.06-0.55$ & $0.65-0.92$ & $100 \%$ \\
\hline & vh & 1 & 0.77 & 0.73 & $1 \%$ & 5 & $0.00-0.64$ & $0.74-0.92$ & $84-100 \%$ \\
\hline \multirow[t]{3}{*}{ Visual-haptic condition } & $\mathrm{v}$ & 1 & 0.91 & 0.86 & $100 \%$ & 5 & $0.02-0.28$ & $0.73-0.87$ & $100 \%$ \\
\hline & $\mathrm{h}$ & 0 & - & - & - & 6 & $0.03-0.67$ & $0.74-0.91$ & $100 \%$ \\
\hline & $\mathrm{vh}$ & 1 & 0.83 & 0.76 & $100 \%$ & 5 & $0.00-0.47$ & $0.77-0.87$ & $100 \%$ \\
\hline
\end{tabular}

Note. Model decision was based on $\mathrm{w}_{\mathrm{i}}(\mathrm{AICc}) . \mathrm{v}=$ visual, $\mathrm{h}=$ haptic, $\mathrm{vh}=$ visual-haptic.

a There is a total of 6 subjects in each first condition group.

b $\mathrm{R}^{2}$ coefficient of determination. 


\section{Experiment 3}

The effect of the first sensory experience with unfamiliar textures on visual roughness perception reported in the first and second experiments is tested here in a setting in which participants are not forced to integrate visual and haptic roughness information. This is different from individuals who performed the bimodal condition last in experiments 1 and 2, in the sense that subjects in the former experiments knew that a combination of visual and haptic texture information in a single bimodal judgment was required. We propose that the order effect of modality conditions on visual roughness perception is primarily expected when subjects are required to combine both kinds of unimodal information to a bimodal judgment, increasing the chance of using haptic imagery for visual roughness estimation. In a setting in which haptic and visual information is processed separately only, we hypothesize that subjects will utilize the higher visual spatial resolution to judge visual roughness.

\subsection{Method}

\subsubsection{Participants}

Twenty-two paid volunteers, four males and 18 females, mean age 23.1 years, participated in this study. Twenty subjects were right handed.

\subsubsection{Stimuli and apparatus}

The set of stimuli consisted of fifteen textures, ranging from 1.50 to $8.50 \mathrm{~mm}$ inter-dot spacing, increasing in steps of $0.50 \mathrm{~mm}$. Visual stimuli were again computer-rendered images of the real textures, presented on a monitor.

\subsubsection{Procedure}

Subjects performed the same roughness estimation task as described in experiment 1 in a visual and haptic condition only. The order of conditions was counter-balanced across subjects and all textures were presented three times in each condition. The practice trials included three textures with an inter-dot distance of 1.50, 5.00 and $8.50 \mathrm{~mm}$ presented in ascending order.

\subsubsection{Data analysis}

Roughness scores were analyzed with a mixed-design ANOVA with the within-subject factors modality (2) and inter-dot spacing (15) and the between-subject factor first modality condition (2). The Greenhouse-Geisser correction was used where appropriate. In addition, a linear and bi-exponential function was fitted to the data as described in experiment 1 . Both models were compared for each subject and condition with the corrected version of the Akaike Information Criterion.

\subsection{Results}

The plot of the mean roughness judgments averaged across both first condition groups (see Fig. 7(a)) indicates attenuated roughness judgments in the visual as compared to the haptic condition. The mixed-design ANOVA of roughness judgments confirms this with significant main effects of the within-subject factors modality, $F(1$, $20)=5.18, p<0.05$, partial $\eta^{2}=0.21$ and inter-dot spacing, $F(1.48$, $29.63)=9.99, p<0.005$, partial $\eta^{2}=0.33$. There is also a significant main effect of the between-subject factor first condition, $F(1,20)=$ $4.56, p<0.05$, partial $\eta^{2}=0.19$, with subjects judging the textures on average less rough when they experienced the stimuli first by touch as compared to vision (see Fig. 7(b)).

Next to the main effects both two-way interactions of First Condition $\times$ Inter-Dot Spacing, $F(1.48,29.63)=5.14, p<0.05$, partial $\eta^{2}=0.20$, and Sensory Modality $\times$ Inter-Dot Spacing, $F(1.96,39.13)=$ $8.95, p<0.005$, partial $\eta^{2}=0.31$ reached significance. These interactions

\section{(a) Average Across Both First-Condition Groups}

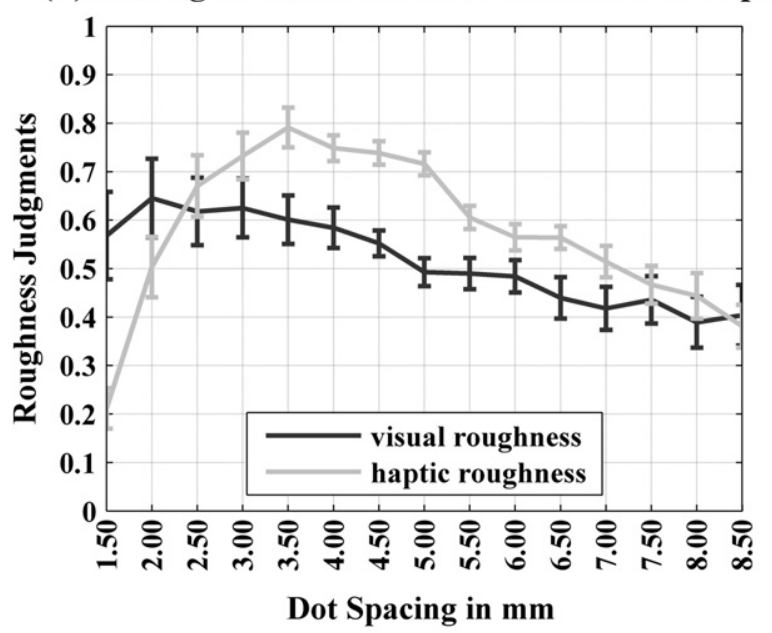

(b) Average Across Both Modalities

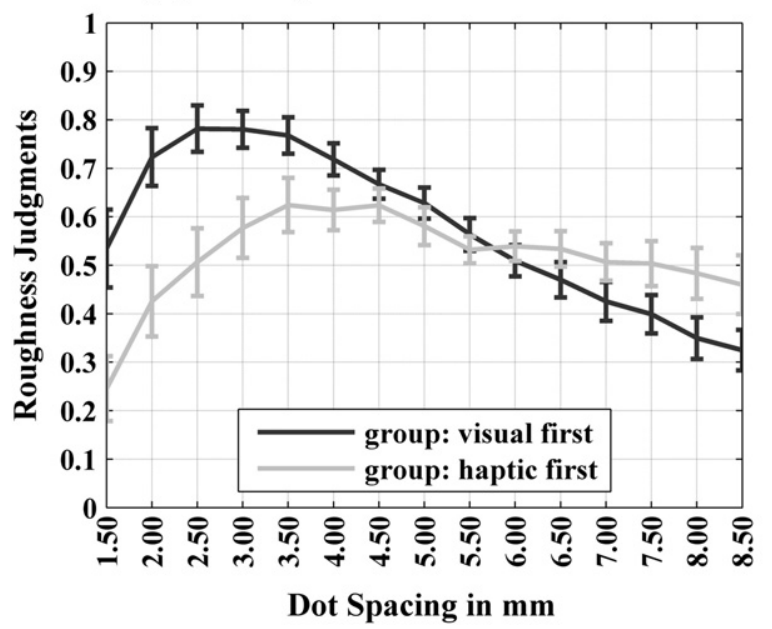

Fig. 7. Mean roughness judgments in Experiment 3, averaged across both first condition groups (a) and averaged across both modality conditions (b). Bars represent the standard error of the mean (SEM).

were further explored by pooling all roughness judgments of textures with inter-dot spacings $\leq 3 \mathrm{~mm}$ and dot distances $>3 \mathrm{~mm}$. Within these groups of inter-dot spacings post hoc contrasts compared roughness estimations of both modality conditions independent of the first condition factor. The results of the post-hoc tests confirmed what is implied by Fig. 7(a), namely significantly lower visual roughness judgments for textures with big inter-dot spacings, mean difference: $-1.25, p<0.005$ and no significant difference for small inter-dot spacings. The comparison of both first condition groups independent of the modality condition yielded a significant result for small inter-dot spacings, mean difference: $2.13, p<0.01$, but not for textures with dot distances $>3 \mathrm{~mm}$ (Fig. 7(b)).

Only the three-way interaction of Modality $\times$ First Condition $\times$ Inter-Dot Spacing did not reach significance in the mixed-design ANOVA, $F(1.96,39.13)=1.82, p=0.18$, partial $\eta^{2}=0.08$.

In line with the roughness estimation task reported in the first two experiments we found the bi-exponential model to be superior to the linear model in the haptic condition, i.e. roughness estimations of all subjects were better characterized by a bi-exponential function of inter-dot spacing. Subjects also reported for the haptic task that textures with very small and very wide inter-element spacing felt smooth while the intermediate surfaces felt rather rough. In contrast, in the visual condition, the results indicated that in 16 out of 22 participants visually perceived roughness was linearly related to 
inter-dot spacing, while only six subjects showed a better fit for the bi-exponential model, with three having experienced the textures first by touch and three in the visual condition. The superiority of the linear model for the visual condition was also reflected in the verbal reports of subjects. Eighteen out of the 22 participants stated that their visual roughness perception was correlated with spatial density or the number of elements on the stimuli. Seven of these subjects had experienced the textures first by touch and 11 first by vision. The three subjects that showed a better fit for the bi-exponential model in the visual condition and experienced the textures first by touch reported that they used the same rating strategy as in the haptic task. One participant could not verbalize the roughness rating strategy used. Table 3 summarizes the results of fitting both models to the data of each subject in both conditions.

\subsection{Discussion}

The analysis of the perceived roughness scores shows that individuals judge textures to be less rough when there is only visual information available, confirming the findings of the first experiment. The curve fitting procedure for the haptic roughness judgments essentially replicates the results of the first two experiments; roughness perception of unfamiliar dot pattern stimuli can be best described as a bi-exponential function of inter-dot distance peaking for textures with about 3-4 mm mean center-to-center dot spacing. However, visual roughness perception was for most subjects linearly related to inter-element spacing, regardless of the first sensory experience with the stimulus material. It is possible that subjects tried to imagine how the textures would feel. However, due to their inexperience with the dot patterns in the visual-first group, participants might have failed to consider the likely decrease of skin deformation (and hence the decrease in roughness) for textures with very small dot spacings, too small for the folds of the skin to enter and to be maximally indented. This would also explain the higher incidence of the linear model in the visual-first groups in experiments 1 and 2. For subjects who experienced the textures first by touch, the haptic imagery process would lead to a bi-exponential roughness function in the visual condition, due to the refined haptic texture concept for this group of participants. However, the results contradict that explanation; an equally high number of subjects who first experienced the textures by touch also showed a better fit for the linear model in the visual condition of the current experiment. This indicates that haptic experience with the stimulus material has only a small or even negligible influence on visual roughness perception of unfamiliar dot pattern textures when no combination of visual and haptic information in a bimodal roughness estimate is necessary. Individuals possibly used the high spatial resolution of the visual sense to estimate roughness of unfamiliar dot pattern surfaces irrespective of previous experience with the textures. The verbal reports of almost all participants, stating that they used the number of elements and spatial density of the textures in the visual condition, further support this hypothesis. However, the fact that not all subjects showed the linear psychophysical roughness function in the visual condition implies that there are also individual differences in the bias toward using one sense or the other, as discussed by Guest and Spence (2003b) in the context of multisensory texture processing. It is conceivable that some subjects used haptic imagery in the visual condition to derive roughness information, resulting in a bi-exponential roughness function of inter-dot spacing just as in the haptic condition. Nevertheless, a consistent roughness concept between modalities is not necessarily helpful in a setting which does not require the combination of both sensory cues.

\section{Experiment 4}

As already discussed before, visual spatial resolution is higher than haptic spatial resolution, possibly explaining why visual roughness estimations still increase for small inter-dot spacings while haptic roughness judgments decrease. This raises the question whether visual roughness estimations decrease again for textures with very small mean center-to-center dot distances, basically representing a similar bi-exponential roughness function of inter-element spacing as in the haptic modality but with a shifted peak toward a smaller spacing (see Fig. 8 for a schematic representation). Hence, in the current experiment we tested the hypothesis that visual dot pattern stimuli with an inter-dot spacing below $1.50 \mathrm{~mm}$ result in a decrease in visually perceived roughness.

\subsection{Methods}

\subsubsection{Participants}

Sixteen subjects, six males and 10 females with a mean age of 34.8 participated in the study.

\subsubsection{Stimuli and apparatus}

Five new stimuli were created with a mean center-to-center dot spacing of $0.25,0.50,0.75,1.00$ and $1.25 \mathrm{~mm}$, resulting in an overlap of neighboring dots and hence in finer textures. In total 34 dot pattern stimuli were presented ranging from 0.25 to $8.50 \mathrm{~mm}$ inter-dot distance and increasing in steps of $0.25 \mathrm{~mm}$.

\subsubsection{Procedure}

Subjects performed the same roughness estimation task as described in experiment 1 but in a visual condition only. All textures were presented four times and the practice run consisted of five example stimuli with an inter-dot spacing of 0.25, 2.25, 4.25, 6.25 and $8.50 \mathrm{~mm}$.

\subsubsection{Data analysis}

A linear and bi-exponential function was fitted to the data and both models were compared with AICc as described in experiment 1 .

\subsection{Results}

Visually perceived roughness averaged over all participants follows an almost identical bi-exponential function as depicted for

Table 3

Summary of the model comparisons of the linear and bi-exponential model for all subjects and conditions (Experiment 3).

\begin{tabular}{|c|c|c|c|c|c|c|c|c|c|}
\hline & \multirow{2}{*}{$\begin{array}{l}\text { First } \\
\text { condition }\end{array}$} & \multicolumn{4}{|c|}{ Linear model selected } & \multicolumn{4}{|c|}{ Bi-exponential model selected } \\
\hline & & $\begin{array}{l}\text { No. of } \\
\text { subjects }^{a}\end{array}$ & $\begin{array}{l}\text { Range of } \mathrm{R}^{2} \\
\text { linear }^{\mathrm{b}}\end{array}$ & $\begin{array}{l}\text { Range of } \mathrm{R}^{2} \\
\text { bi-exponential }^{\mathrm{b}}\end{array}$ & $\begin{array}{l}\text { Range of } \mathrm{w}_{\mathrm{i}}(\mathrm{AICc}) \\
\text { bi-exponential }\end{array}$ & $\begin{array}{l}\text { No. of } \\
\text { subjects }^{a}\end{array}$ & $\begin{array}{l}\text { Range of } \mathrm{R}^{2} \\
\text { linear }^{\mathrm{b}}\end{array}$ & $\begin{array}{l}\text { Range of } \mathrm{R}^{2} \\
\text { bi-exponential }^{\mathrm{b}}\end{array}$ & $\begin{array}{l}\text { Range of } \mathrm{w}_{\mathrm{i}} \text { (AICc) } \\
\text { bi-exponential }\end{array}$ \\
\hline \multirow[t]{2}{*}{ Visual condition } & $\mathrm{v}$ & 8 & $0.84-0.96$ & $0.80-0.96$ & $0-46 \%$ & 3 & $0.07-0.92$ & $0.51-0.96$ & $61-93 \%$ \\
\hline & $\mathrm{h}$ & 8 & $0.86-0.97$ & $0.84-0.97$ & $0-17 \%$ & 3 & $0.03-0.37$ & $0.52-0.89$ & $74-100 \%$ \\
\hline \multirow[t]{2}{*}{ Haptic condition } & $\mathrm{v}$ & 0 & - & - & - & 11 & $0.00-0.73$ & $0.67-0.99$ & $80-100 \%$ \\
\hline & $\mathrm{h}$ & 0 & - & - & - & 11 & $0.00-0.92$ & $0.51-0.97$ & $60-100 \%$ \\
\hline
\end{tabular}

Note. Model decision was based on $\mathrm{w}_{\mathrm{i}}(\mathrm{AICc}) . \mathrm{v}=$ visual, $\mathrm{h}=$ haptic.

a There is a total of 11 subjects in each first condition group.

b $\mathrm{R}^{2}$ coefficient of determination. 


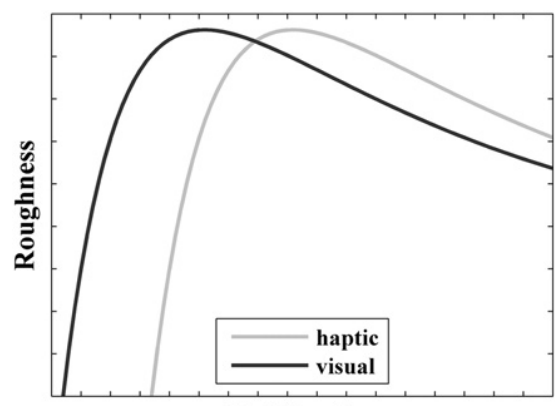

Dot Spacing

Fig. 8. Model of the psychophysical function representing visual compared to haptic roughness perception.

roughness experienced by touch in the previous experiments (see Fig. 9). The only difference to the haptic function is an earlier peak for textures with an inter-dot spacing of about 1.25-1.75 mm.

The superiority of the bi-exponential model for this set of visual textures was confirmed by the number of subjects who showed a better fit for the bi-exponential compared to the linear curve, i.e. 15 versus 1 (see Table 4 ).

\subsection{Discussion}

The results of this experiment show that visual roughness perception of unfamiliar dot pattern stimuli ranging from 0.25 to $8.50 \mathrm{~mm}$ inter-element spacing is well characterized by a bi-exponential function peaking for textures with an inter-dot spacing of about 1.25$1.75 \mathrm{~mm}$. This indicates that the haptic and visual psychophysical function for roughness perception of unfamiliar dot patterns can be characterized by the same curve but differs in the location of the maximum, likely due to a difference in the spatial resolving power between both modalities. We do not claim that spatial resolution is the only factor limiting visual roughness perception of these textures. Certainly, contrast sensitivity also contributes to a decline in roughness perception, especially for textures below $0.75 \mathrm{~mm}$ inter-dot spacing for which a big overlap between texture elements is present and hence a decrease in local contrast. However, visual roughness perception seems to decrease already for textures with an inter-dot spacing of 0.75 and $1.00 \mathrm{~mm}$ for which contrast sensitivity as the limiting factor seems rather unlikely.

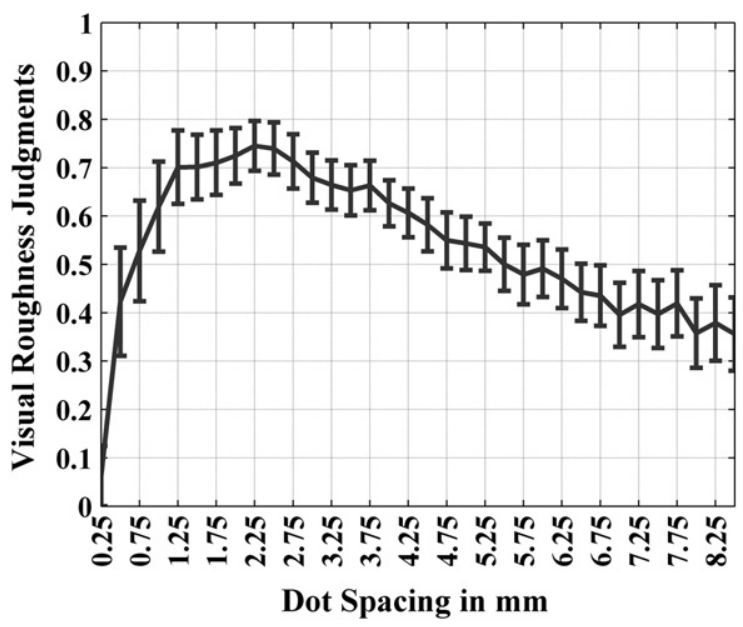

Fig. 9. Mean visual roughness judgments in Experiment 4. Bars represent the standard error of the mean (SEM).
Table 4

Summary of the model comparisons of the linear and bi-exponential model for visually perceived roughness of all subjects (Experiment 4).

\begin{tabular}{lclll}
\hline $\begin{array}{l}\text { Model } \\
\text { selected }\end{array}$ & $\begin{array}{l}\text { No. of } \\
\text { subjects }^{\mathrm{a}}\end{array}$ & $\begin{array}{l}\text { Range of }^{2} \\
\text { linear }^{\mathrm{b}}\end{array}$ & $\begin{array}{l}\text { Range of } \mathrm{R}^{2} \\
\text { bi-exponential }^{\mathrm{b}}\end{array}$ & $\begin{array}{l}\text { Range of } \mathrm{w}_{\mathrm{i}} \text { (AICc) } \\
\text { bi-exponential }\end{array}$ \\
\hline $\begin{array}{l}\text { Linear } \\
\text { Bi-exponential }\end{array}$ & 15 & 0.98 & 0.98 & $31 \%$ \\
\hline $\begin{array}{l}\text { Note. Model decision was based on } \mathrm{w}_{\mathrm{i}} \text { (AICc). } \\
\text { a } \text { There is a total of } 16 \text { subjects. }\end{array}$ \\
b $\mathrm{R}^{2}$ coefficient of determination.
\end{tabular}

\section{Experiment 5}

Note that there is another explanation for the results in experiment 4; due to a missing scale reference for size comparison of the visual stimuli, subjects might have judged the size of the textures differently than they would have in a natural haptic roughness exploration condition, resulting in a different peak location of the visual roughness function. Therefore, we presented the same textures again to a new group of observers, but this time in four different scale reference conditions, in order to test the alternative hypothesis that visual roughness perception of dot pattern textures is influenced by the scale context when stimuli are presented on a monitor.

\subsection{Methods}

\subsubsection{Participants}

Twelve subjects, five males and seven females with a mean age of 21.5 participated in the study.

\subsubsection{Stimuli and apparatus}

The stimulus set was the same as in experiment 4. However, in addition to a no-reference condition in which no scale reference was presented, there were three other conditions showing a picture of a hand next to the textures in varying sizes, i.e. with a natural size ratio of hand and stimulus, a hand with $50 \%$ and a hand with $150 \%$ of the natural size ratio (see Fig. 10).

\subsubsection{Procedure}

Subjects performed the same visual roughness estimation task as described in experiment 4. Participants were informed that they would be presented with one texture at a time and were asked to evaluate the roughness of this texture. No specific instruction on how to use the reference picture next to the texture was provided. The order of conditions was counter-balanced across subjects and all textures were presented randomly, three times in each condition. The practice run, in the beginning of the experiment, consisted of five example stimuli with an inter-dot spacing of $0.25,2.25,4.25,6.25$ and $8.50 \mathrm{~mm}$ and no scale reference.

\subsubsection{Data analysis}

Roughness scores were analyzed with a repeated measures ANOVA with the within-subject factors scale reference condition (4) and inter-dot spacing (34). The Greenhouse-Geisser adjustment for degrees of freedom was used when the sphericity assumption was violated. Additionally, a linear and bi-exponential function was fitted to the data and both models were compared separately for all four scale reference conditions with AICc.

\subsection{Results}

The repeated measures ANOVA showed only a main effect of the within-subject factor inter-dot spacing $F(1.74,19.16)=14.36$, $p<0.001$, partial $\eta^{2}=0.57$. Neither the main effect of the factor scale reference nor the Scale Reference $\times$ Inter-Dot Spacing interaction 

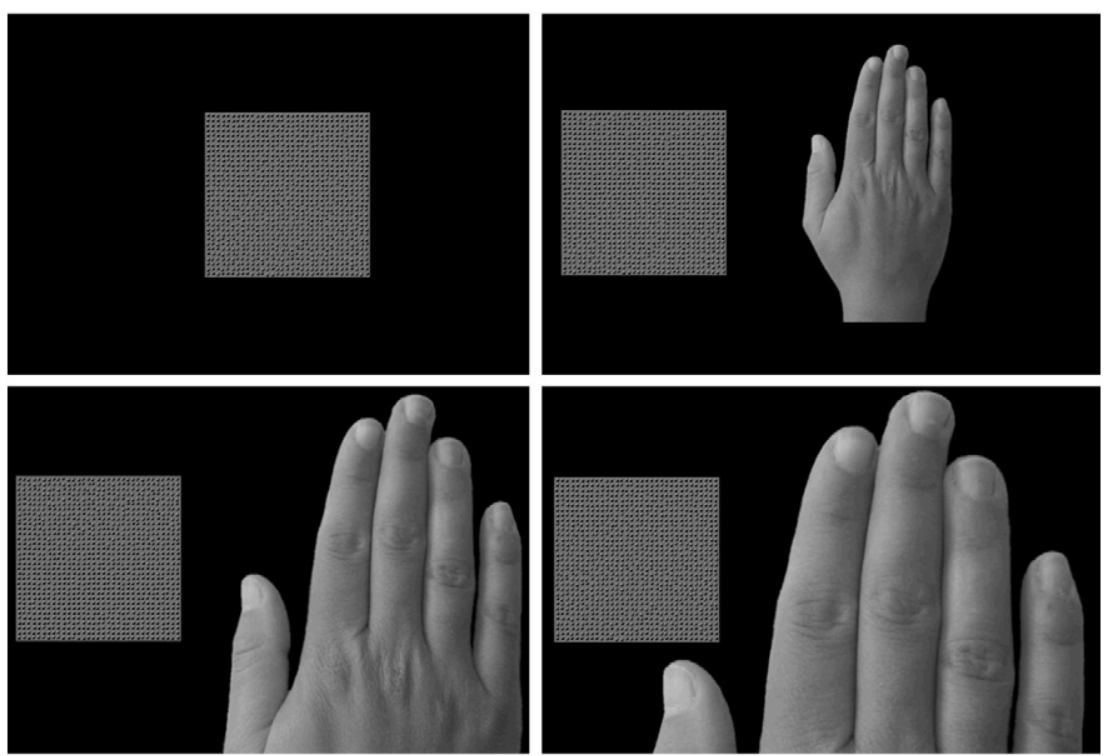

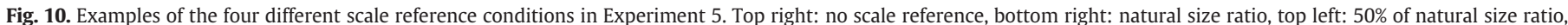
bottom left: $150 \%$ of natural size ratio.

reached significance, $F(2.35,25.87)=0.61, p=0.58$, partial $\eta^{2}=0.05$ and $F(4.79,52.71)=1.03, p=0.41$, partial $\eta^{2}=0.09$, respectively.

The model fitting confirms the results of the ANOVA showing that there is no effect of scale context on visual roughness perception in that experiment (see Fig. 11). All 12 subjects showed a better fit for the bi-exponential as compared to the linear model in all four scale reference conditions (see Table 5).

\subsection{Discussion}

The results of the fifth experiment indicate that visual roughness perception of dot pattern textures is invariant to changes in scale context when stimuli are presented on a monitor. Visual roughness perception of these unfamiliar textures was well described by a bi-exponential function peaking at about 1.25-1.75 mm inter-dot spacing regardless of the scale context in which the textures were presented. We are aware that we tested only a limited subset of scale contexts and that by choosing more extreme size differences between textures and the hand, the results might have been different. However, this experiment aimed at investigating whether size miscalculations of the virtual visual stimuli in comparison to the real

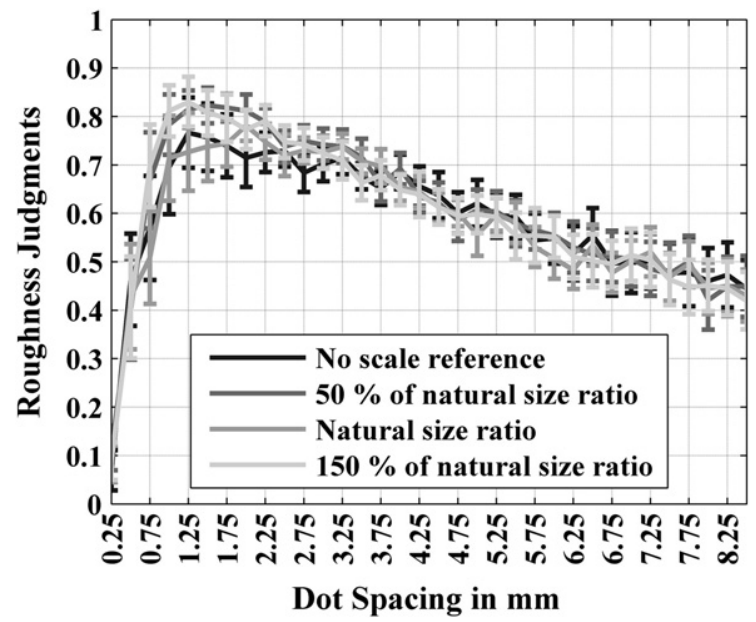

Fig. 11. Mean visual roughness judgments for all four scale reference conditions in Experiment 5. Bars represent the standard error of the mean (SEM). textures might have resulted in the change of the maximum of the visual roughness function toward smaller inter-dot spacings in experiment 4 . By testing these naturalistic size differences between the textures and the hand, we believe we were able to show that this alternative explanation is rather unlikely. Therefore, our initial interpretation of the findings seems to hold, i.e. the difference in the peak location of the visual and haptic roughness function of dot pattern textures can be well explained by the better spatial resolution of the visual system.

\section{General discussion}

In the experiments reported in this paper we tested whether roughness perception shows a linear or an inverted U-shape relationship with inter-element spacing of dot patterns, and whether this relationship is different for haptic, visual or combined visual-haptic input. We found an effect of sensory modality on roughness perception. The relationship of inter-element spacing and roughness perceived by touch, by vision as well as by vision and touch combined was best described by a bi-exponential psychophysical function. Haptic and visual-haptic roughness perception peaked between 3 and $4 \mathrm{~mm}$ and were in the same order of magnitude. In contrast, visual roughness peaked already at about $1.25 \mathrm{~mm}$ inter-element spacing when tested in isolation, and this effect was invariant to differences in the scale context. Moreover, lower visual than haptic and bimodal roughness scores were found, primarily for textures with inter-dot spacings above $3 \mathrm{~mm}$. Furthermore, the order of modality conditions affected the visual roughness function in a setting in which subjects were asked to combine both visual and haptic information in a single bimodal judgment. There was a trend towards greater similarity between visual and haptic and visualhaptic roughness perception in terms of peak location when haptic preceded visual experience with the dot pattern textures. This influence of sensory familiarity on visual roughness scores was not replicated in a context where subjects were merely asked to perform unimodal visual and haptic roughness estimations. By comparing results obtained by using real and virtual visual textures, we were able to show that the main findings do not change in an experimental context with restricted viewing conditions.

In the first three experiments haptically perceived roughness was characterized by an increase up to an inter-dot spacing of about $3 \mathrm{~mm}$, decreasing again after a short plateau. We speculate that the number of indentations of the skin as well as the local spatial 
Table 5

Summary of the model comparisons of the linear and bi-exponential model for all subjects and conditions (Experiment 5).

\begin{tabular}{|c|c|c|c|c|c|c|c|c|}
\hline & \multicolumn{4}{|c|}{ Linear model selected } & \multicolumn{4}{|c|}{ Bi-exponential model selected } \\
\hline & $\begin{array}{l}\text { No. of } \\
\text { subjects }^{\text {a }}\end{array}$ & $\begin{array}{l}\text { Range of } \mathrm{R}^{2} \\
\text { linear }^{\mathrm{b}}\end{array}$ & $\begin{array}{l}\text { Range of } \mathrm{R}^{2} \\
\text { bi-exponential }^{\mathrm{b}}\end{array}$ & $\begin{array}{l}\text { Range of } \mathrm{w}_{\mathrm{i}}(\mathrm{AICc}) \\
\text { bi-exponential }\end{array}$ & $\begin{array}{l}\text { No. of } \\
\text { subjects }^{\text {a }}\end{array}$ & $\begin{array}{l}\text { Range of } \mathrm{R}^{2} \\
\text { linear }^{\mathrm{b}}\end{array}$ & $\begin{array}{l}\text { Range of } \mathrm{R}^{2} \\
\text { bi-exponential }^{\mathrm{b}}\end{array}$ & $\begin{array}{l}\text { Range of } w_{i}(\mathrm{AICc}) \\
\text { bi-exponential }\end{array}$ \\
\hline No scale reference & 0 & - & - & - & 12 & $0.01-0.83$ & $0.42-0.95$ & $100 \%$ \\
\hline Natural size ratio & 0 & - & - & - & 12 & $0.00-0.76$ & $0.51-0.97$ & $100 \%$ \\
\hline $150 \%$ of natural size ratio & 0 & - & - & - & 12 & $0.04-0.88$ & $0.76-0.96$ & $100 \%$ \\
\hline $50 \%$ of natural size ratio & 0 & - & - & - & 12 & $0.00-0.76$ & $0.62-0.97$ & $100 \%$ \\
\hline
\end{tabular}

Note. Model decision was based on $\mathrm{w}_{\mathrm{i}}$ (AICc).

a There is a total of 12 subjects.

b $\mathrm{R}^{2}$ coefficient of determination.

displacement (i.e., the depth of indentations) caused by the single dots influence the haptic roughness function. More specifically, for widely spaced textures the number of indentations is low while the local spatial displacement is maximal. If dots are added to the texture, the number of indentations will increase, thus increasing the perceived roughness. Below a certain dot distance, the local spatial displacement of the skin as well as the ability to resolve the single texture elements will be increasingly restricted by the skin's (lack of) compliance, thus the resulting roughness percept will decrease again.

In the first and third experiments we found attenuated roughness scores in the visual compared to the haptic and bimodal condition, which was not reported in a study conducted by Lederman and Abbott (1981) and which we also failed to find in our second study with a natural visual condition. There are several explanations for the difference in the results. One explanation might be the differences in presentation mode of the stimuli. Lederman and Abbott (1981) presented the textures in their natural form in the visual and bimodal condition, as we did in our second experiment, while we presented the textures on a monitor for visual exploration in experiments 1 and 3 . This change of presentation mode between the haptic and visual condition in our experiments 1 and 3 might have induced or enhanced differences in roughness estimations between the conditions. However, the different findings in both studies can also be explained by the macrospatial structure of the textures that we used. The difference in perceived roughness magnitude between the visual condition and settings in which also haptic information was available is a logical finding for unfamiliar dot pattern stimuli when taking the results of experiments 3-5 into account. Our results suggest that visual and haptic roughness perception are both well described by a bi-exponential function of inter-dot spacing, shifted toward smaller spacings for visual exploration probably due to the higher visual spatial resolution. This shift results in attenuated visual roughness scores for textures above approximately $3 \mathrm{~mm}$ dot distance, textures that were overrepresented in our set of stimuli, and in higher visual roughness scores for textures with smaller inter-dot spacings. The interaction of modality and spacing was found in all 3 of our experiments containing haptic roughness estimation conditions. One explanation for the missing main effect of modality in the second experiment is the rather small effect of attenuated visual roughness scores for inter-element spacings above $3 \mathrm{~mm}$. This attenuation might have been cancelled out by the rather strong difference between visual and haptic roughness judgments for smaller inter-dot spacings. Finally, another explanation could be the influence of the task demands and haptic stimulus familiarity, which are found to affect visual roughness estimation strategies in the current study. The significant interaction of sensory modality, first condition and inter-dot distance in both experiments containing bimodal conditions indicates that the relationship of dot spacing and roughness estimation differs not only with the kind of sensory information available but it is also modulated by the kind of previous experience with the stimuli. An alternative explanation for the conflicting findings might therefore be a difference in the familiarity with the stimulus material. Lederman and Abbott (1981) used abrasive paper which is familiar to most people and therefore the visual judgments might have been already influenced by the haptic experience with those textures, diminishing the difference between visual and haptic magnitude estimates. Furthermore, subjects were allowed to practice for one day and therefore to become well acquainted with all stimuli. In contrast, in our experiment subjects had only few practice trials before each condition started. Hence, the factor haptic familiarity might have influenced roughness perception. This hypothesis is further supported by the results of the curve fitting procedure in the first and second experiment. The relationship of roughness perception and inter-dot spacing in experiments 1 and 2 is clearly influenced by the sensory information available for the judgments but also by previous experience with the stimulus material. When combined visual-haptic roughness judgments are required people appear to use preferably haptic imagery strategies to estimate visual roughness and therefore to calibrate the visual estimates to haptic roughness perception. In a setting in which extensive haptic experience with the stimulus material is available, this might result in a complete elimination of visual and haptic differences, which would be consistent with the findings from Lederman and Abbott (1981) for finer textures.

Several individuals reported that they used haptic imagery for visual roughness estimation when bimodal judgments were required and others utilized the high spatial resolution of the visual sense for roughness estimation when a combination of the unimodal information was not necessary. Still, some variation in the applied strategies and hence in the psychophysical roughness functions remained. This indicates an effect of individual biases toward the use of haptic or visual information in texture perception, as discussed by Guest and Spence (2003b) in the context of a multisensory task. Nevertheless, the results of our first and second experiments clearly indicate that bimodal roughness perception of unfamiliar dot pattern stimuli is more influenced by haptic than by visual information, in line with previous findings (Heller, 1982; Lederman et al., 1986). It is noteworthy, however, that there are also alternative explanations for the close resemblance of the bimodal and haptic roughness judgments in the work presented here. Although the experimenter monitored the performance of the participants in all conditions, it is possible that subjects focused their attention mainly on the haptic exploration in the bimodal condition and neglected the visual exploration of the textures. This would result necessarily in very similar haptic and bimodal roughness estimates. However, when asked to describe their rating strategies in the debriefing phase, none of our participants reported to have completely neglected the visual information in the visual-haptic condition. There is, therefore, every indication that subjects indeed performed the visual exploration as instructed. The use of an eyetracker system in future studies might be valuable for controlling the visual exploration behavior in the bimodal condition, although one can still not be certain whether the visual information is actually taken into account for bimodal judgments.

\section{Conclusions}

We reported the findings of a series of five experiments investigating the effect of sensory modality and familiarity on perceived 
roughness of unfamiliar textures. Visual, haptic and bimodal visualhaptic roughness perception of unfamiliar dot pattern stimuli is best characterized by a bi-exponential function of inter-dot spacing. However, the sensory modality used to explore the textures has an effect on the perceived magnitude of roughness. This is likely due to the difference in spatial resolution between the haptic and visual modality, resulting in a shift of the peak of the bi-exponential visual roughness function toward higher spatial resolution textures. Moreover, individuals appear to adapt their visual roughness estimation strategies based on the task demands, namely whether bimodal or only unimodal evaluations are required and on the sensory familiarity with the stimulus material. For the set of stimuli employed, a bias toward haptic information was demonstrated for bimodal visual-haptic roughness estimations. The overall results were independent of the presentation mode of the visual textures (virtual vs. real textures), indicating the suitability of the experimental design for neuroimaging studies with restricted viewing conditions and allowing to establish a more direct link between behavioral and neuroimaging results.

\section{Acknowledgments}

The research leading to these results has received funding from the European Community's Seventh Framework Programme FP7/ 2007-2013 under grant agreement number PITN-GA-2008-214728. Rainer Goebel and Amanda Kaas also gratefully acknowledge the support of the BrainGain Smart Mix Programme of the Netherlands Ministry of Economic Affairs and the Netherlands Ministry of Education, Culture and Science.

We would like to thank Ron Hellenbrand for his help in designing the stimulus material. We are also grateful to Minye Zhan for her assistance with the data acquisition and to Dr. Hong Z. Tan for the very helpful remarks on the results of the first experiment.

\section{References}

Bensmaïa, S., \& Hollins, M. (2005). Pacinian representations of fine surface texture. Perception \& Psychophysics, 67, 842-854.

Bergmann Tiest, W. M. (2010). Tactual perception of material properties. Vision Research, 50, 2775-2782.

Bergmann Tiest, W. M., \& Kappers, A. M. L. (2006). Analysis of haptic perception of materials by multidimensional scaling and physical measurements of roughness and compressibility. Acta Psychologica, 121, 1-20.

Bergmann Tiest, W. M., \& Kappers, A. M. L. (2007). Haptic and visual perception of roughness. Acta Psychologica, 124, 177-189.

Blake, D. T., Hsiao, S. S., \& Johnson, K. O. (1997). Neural coding mechanisms in tactile pattern recognition: The relative contributions of slowly and rapidly adapting mechanoreceptors to perceived roughness. The Journal of Neuroscience, 17, 7480-7489.

Burnham, K. P., \& Anderson, D. R. (2004). Multimodel inference: Understanding AIC and BIC in model selection. Sociological Methods \& Research, 33, 261-304.

Cascio, C. J., \& Sathian, K. (2001). Temporal cues contribute to tactile perception of roughness. The Journal of Neuroscience, 21, 5289-5296.

Connor, C. E., Hsiao, S. S., Phillips, J. R., \& Johnson, K. O. (1990). Tactile roughness: Neural codes that account for psychophysical magnitude estimates. The Journal of Neuroscience, 10, 3823-3836.

Dépeault, A., Meftah, E. M., \& Chapman, C. E. (2009). Tactile perception of roughness: Raised-dot spacing, density and disposition. Experimental Brain Research, 197, 235-244.
Gescheider, G. A., Bolanowski, S. J., Greenfield, T. C., \& Brunette, K. E. (2005). Perception of the tactile texture of raised-dot patterns: A multidimensional analysis. Somatosensory and Motor Research, 22, 127-140.

Guest, S., Catmur, C., Lloyd, D., \& Spence, C. (2002). Audiotactile interactions in roughness perception. Experimental Brain Research, 146, 161-171.

Guest, S., \& Spence, C. (2003a). Tactile dominance in speeded discrimination of textures. Experimental Brain Research, 150, 201-207.

Guest, S., \& Spence, C. (2003b). What role does multisensory integration play in the visuotactile perception of texture? International Journal of Psychophysiology, 50, 63-80.

Helbig, H. B., \& Ernst, M. O. (2007a). Knowledge about a common source can promote visual-haptic integration. Perception, 36, 1523-1533.

Helbig, H. B., \& Ernst, M. O. (2007b). Optimal integration of shape information from vision and touch. Experimental Brain Research, 179, 595-606.

Heller, M. A. (1982). Visual and tactual texture perception: Intersensory cooperation. Perception \& Psychophysics, 31, 339-344.

Heller, M. A. (1989). Texture perception in sighted and blind observers. Perception $\mathcal{E}$ Psychophysics, 45, 49-54.

Ho, Y., Landy, M. S., \& Maloney, L. T. (2006). How direction of illumination affects visually perceived surface roughness. Journal of Vision, 6, 634-648.

Ho, Y., Maloney, L. T., \& Landy, M. S. (2007). The effect of viewpoint on perceived visual roughness. Journal of Vision, 7, 1-16.

Hollins, M., \& Risner, S. R. (2000). Evidence for the duplex theory of tactile texture perception. Perception \& Psychophysics, 62, 695-705.

Jones, B., \& O'Neil, S. (1985). Combining vision and touch in texture perception. Perception Er Psychophysics, 37, 66-72.

Kahrimanovic, M., Bergmann Tiest, W. M., \& Kappers, A. M. L. (2009). Context effects in haptic perception of roughness. Experimental Brain Research, 194, 287-297.

Katz, D. (1989). The world of touch. Hillsdale, England: Lawrence Erlbaum Associates, Inc (Original work published in 1925.).

Klatzky, R. L., \& Lederman, S. J. (1999). Tactile roughness perception with a rigid link interposed between skin surface. Perception \& Psychophysics, 61, 591-607.

Klatzky, R. L., Lederman, S. J., \& Reed, C. (1987). There's more to touch than meets the eye: The salience of object attributes for haptics with and without vision. Journal of Experimental Psychology, 116, 356-369.

Lacey, S., Hall, J., \& Sathian, K. (2010). Are surface properties integrated into visuohaptic object representations? European Journal of Neuroscience, 31, 1882-1888.

Lawrence, M. A., Kitada, R., Klatzky, R. L., \& Lederman, S. J. (2007). Haptic roughness perception of linear gratings via bare finger or rigid probe. Perception, 36, 547-557.

Lederman, S. J., \& Abbott, S. G. (1981). Texture perception: Studies of intersensory organization using a discrepancy paradigm, and visual versus tactual psychophysics. Journal of Experimental Psychology. Human Perception and Performance, 7, 902-915.

Lederman, S. J., \& Klatzky, R. L. (2009). Haptic perception: A tutorial. Attention, Perception, E' Psychophysics, 71, 1439-1459.

Lederman, S. J., Klatzky, R. L., Hamilton, C. L., \& Ramsay, G. I. (1999). Perceiving roughness via a rigid probe: Psychophysical effects of exploration speed and mode of touch. Haptics-e.

Lederman, S. J., Thorne, G., \& Jones, B. (1986). Perception of texture by vision and touch: Multidimensionality and intersensory integration. Journal of Experimental Psychology. Human Perception and Performance, 12, 169-180.

Legge, G. E., Madison, C., Vaughn, B. N., Cheong, A. M., \& Miller, J. C. (2008). Retention of high tactile acuity throughout the life span in blindness. Perception $\mathcal{E}$ Psychophysics, 70, 1471-1488.

Meftah, E. M., Belingard, L., \& Chapman, C. E. (2000). Relative effects of the spatial and temporal characteristics of scanned surfaces on human perception of tactile roughness using passive touch. Experimental Brain Research, 132, 351-361.

Merabet, L. B., Swisher, J. D., McMains, S. A., Halko, M. A., Amedi, A., \& Pascual-Leone, A (2007). Combined activation and deactivation of visual cortex during tactile sensory processing. Journal of Neurophysiology, 97, 1633-1641.

Merabet, L. B., Thut, G., Murray, B., Andrews, J., Hsiao, S., \& Pascual-Leone, A. (2004) Feeling by sight or seeing by touch? Neuron, 42, 173-179.

Sekuler, R., \& Blake, R. (2001). Perception (4th ed.). New York: McGraw-Hill.

Wagenmakers, E., \& Farrell, S. (2004). AIC model selection using Akaike weights. Psychonomic Bulletin \& Review, 11, 192-196.

Yoshioka, T., Bensmaïa, S. J., Craig, J. C., \& Hsiao, S. S. (2007). Texture perception through direct and indirect touch: An analysis of perceptual space for tactile textures in two modes of exploration. Somatosensory and Motor Research, 24, 53-70. 